\title{
Relay Selection for Security Improvement in Cognitive Radio Networks with Energy Harvesting
}

\author{
Khuong Ho-Van $\mathbb{D}^{1,2}$ and Thiem Do-Dac $\mathbb{D}^{1,2,3}$ \\ ${ }^{1}$ Ho Chi Minh City University of Technology (HCMUT), 268 Ly Thuong Kiet Street, District 10, Ho Chi Minh City, Vietnam \\ ${ }^{2}$ Vietnam National University Ho Chi Minh City, Linh Trung Ward, Thu Duc District, Ho Chi Minh City, Vietnam \\ ${ }^{3}$ Thu Dau Mot University, 6 Tran Van On Street, Phu Hoa Ward, Thu Dau Mot City, Binh Duong Province, Vietnam
}

Correspondence should be addressed to Thiem Do-Dac; thiemdd@tdmu.edu.vn

Received 22 March 2021; Revised 20 April 2021; Accepted 13 May 2021; Published 21 June 2021

Academic Editor: Xingwang Li

Copyright @ 2021 Khuong Ho-Van and Thiem Do-Dac. This is an open access article distributed under the Creative Commons Attribution License, which permits unrestricted use, distribution, and reproduction in any medium, provided the original work is properly cited.

\begin{abstract}
This paper selects an unlicensed relay among available self-powered relays to not only remain but also secure information transmission from an unlicensed source to an unlicensed destination. The relays harvest energy in signals of the unlicensed source and the licensed transmitter. Then, they spend the harvested energy for their relaying operation. Conditioned on the licensed outage restriction, the peak transmission power restriction, Rayleigh fading, and the licensed interference, the current paper proposes an exact closed-form formula of the secrecy outage probability to quickly evaluate the secrecy performance of the proposed relay selection method in cognitive radio networks with energy harvesting. The proposed formula is corroborated by computer simulations. Several results illustrate the effectiveness of the relay selection in securing information transmission. Additionally, the security capability is saturated at large peak transmission powers or large preset outage probabilities of licensed users. Furthermore, the security capability depends on many specifications among which the power splitting ratio, the relays' positions, and the time switching ratio can be optimally selected to obtain the best security performance.
\end{abstract}

\section{Introduction}

In cognitive radio networks, unlicensed users are supported to use opportunistically the allotted frequencies of licensed users to improve significantly the spectrum utilization efficiency, which is currently low [1]. Amidst three typical operation mechanisms of cognitive radios (interweave, underlay, overlay), the underlay one has received more attention because of its low system design complexity [2]. Following this trend, our work also considers this mechanism. The underlay mechanism requires the power allotment for unlicensed users strictly subject to the licensed outage constraint (This paper uses "constraint" and "restriction" interchangeably) inflicted by communication reliability of licensed users and the peak transmission power restriction imposed by hardware design [3]. These power constraints bound the transmission power of unlicensed users, inducing insufficient power to reliably transmit information directly from an unlicensed source to an unlicensed destination. Additionally, severe wave propagation conditions (heavy path-loss, strong shadowing, and severe fading) induce communication outage for the direct channel between the unlicensed sourcedestination pair. Therefore, exploiting unlicensed relays in this direct link can significantly mitigate these severe wave propagation conditions and eliminate the need of large transmission power over a wide coverage range. Consequently, the relays can play a role as a bridge for the source information to reach the destination. Instead of exploiting all available relays which either transmit simultaneously in one orthogonal channel or sequentially in several orthogonal channels, selecting one relay among them according to a certain criterion is considered as an efficient-and-economical solution in terms of complexity, power, and bandwidth [4-11]. However, as an assistant, the selected relay may be unwilling to consume its individual energy for assistant activity. Nowadays, advanced technologies allow wireless users to power their operations by the harvested radio frequency (RF) energy [12-15]. As a result, the selected relay can extend 
the coverage range of the unlicensed source with the harvested energy, better maintaining reliable source-todestination communications. Nevertheless, the harvested energy is limited and, thus, the issue is whether the selected relay can ensure secure-and-reliable communication for source data against the eavesdropping of wire-tappers in the viewpoint of information theory. This viewpoint affirms that the positive subtraction of the wire-tap channel capacity from the main channel capacity ensures the secured communications [16]. The current paper solves such an issue.

1.1. Previous Works. While most recent publications focused on securing direct transmission (i.e., without relaying [1723]) and relaying transmission (i.e., without relay selection [24-28]) in cognitive radio networks with energy harvesting (CRNwEH) by the physical layer security technique (e.g., [29-33]), to the best of our understanding only three works in [34-36] mentioned the relay selection in these networks. To be specific, [34] studied the security capability of the conventional reactive relay selection in CRNwEH in terms of the secrecy outage probability (SOP) through Monte-Carlo simulations under the (peak transmission and interference) power constraints. The conventional reactive relay selection selects one relay which produces the highest signal-to-noise ratio (SNR) to the destination as compared to all remaining relays. Additionally, [34] considered the time switching protocol for energy harvesting, which enables the relay to harvest energy from merely the unlicensed source signal. To improve the security capability, [35] suggested a link selection method in which the link ( $\mathrm{A}$ link is a multihop communication link through which the unlicensed source transmits information to the unlicensed destination via multiple unlicensed relays) of the largest capacity is adopted. Also, [35] permitted the relays to scavenge the energy in the beacon signals with the time switching protocol. Furthermore, [35] solely analyzed the connection outage probabilities (The connection outage probability indicates the possibility that the received SNR is smaller than a target value) of the wire-tapper and the unlicensed destination. Recently, ([37] is the conference version of [36]. In [37], a part of the results of [36] was briefly presented without any proof. However, the direct link was considered in [37] but not in [36]) [36] suggested a relay selection method where the adopted relay must successfully recover the unlicensed source's information and minimize the SNR at the wire-tapper. In [36], the (peak transmission and interference) power constraints were imposed on the unlicensed transmission power, and the power splitting protocol was considered for energy harvesting. Additionally, [36] only analyzed the intercept outage probability (The intercept outage probability signifies the possibility that the SNR at the wire-tapper is lower than a target value). In addition, [34-36] neglected the interference from the licensed transmitter (shortly, licensed interference). In general, the licensed interference should be considered in the underlay mechanism because both licensed and unlicensed users transmit on the same wireless channel. Moreover, the licensed interference is useful for energy harvesting at relays. Furthermore, none of [34-36] neither investigated the licensed outage constraint nor analyzed the SOP, a key security capability indicator in the viewpoint of information theory.
1.2. Motivations. This paper extends $[34,36]$ with the following remarkable differences (Since the system models in [34, 36] and ours are completely different, it is impossible to compare their security performance under the same system parameters):

(i) The relays work solely as they are able to recover successfully the unlicensed source's information. Such an operation of the relays avoids the error propagation (e.g., [34])

(ii) The proposed relay selection method (It is obvious that this paper completely differs [35] because the former proposed the relay selection while the latter proposed the link selection) selects one relay which produces the largest maximum secrecy rate (As recommended by the reviewer, "maximum secrecy rate" should be used in this paper instead of "secrecy capacity" which is normally used in the literature) as compared to activated relays. On the contrary, [34] selects one relay which produces the largest SNR to the unlicensed destination as compared to all remaining relays, and [36] selects one relay which produces the smallest SNR to the wire-tapper as compared to activated relays. Therefore, the relay selection method in [34] only concentrated on improving the main (the unlicensed selected relay to the unlicensed destination) channel capacity while that in [36] merely focused on degrading the wire-tap (the unlicensed selected relay to the wiretapper) channel capacity. It is reminded that the subtraction of the wire-tap channel capacity from the main channel capacity is the maximum secrecy rate [16]. Accordingly, our relay selection is more complete than $[34,36]$ in improving and evaluating the security performance in the viewpoint of information theory

(iii) The licensed interference is investigated in analyzing the security performance of the relay selection in $\mathrm{CRNwEH}$ while $[34,36]$ ignored this interference. Considering this interference complicates the performance analysis but valuable because the licensed interference should not be neglected in the underlay mechanism in general

(iv) The relays take advantage of the licensed interference for energy harvesting. Apparently, converting unexpected signals to advantageous energy sources is valuable and this also distinguishes $[34,36]$ where the energy in the licensed interference is not harvested at relays

(v) The power splitting based energy harvesting method is employed in this paper while [34] uses the time switching based energy harvesting method

(vi) Our work suggests the precise closed-form SOP analysis that distinguishes [34] where merely simulation results were provided and [36] where the intercept outage probability (IOP) was analyzed. It 
is reminded that the SOP analysis in this paper is more complete than the IOP analysis in [36] because the former considers both the wire-tap and main channels while the latter only investigated the wire-tap channel. Moreover, our SOP analysis considers the licensed outage constraint while $[34,36]$ ignored it

\subsection{Contributions. Our work contributes the following:}

(i) Propose the relay selection method for securing the unlicensed source-to-destination information transmission in case that their direct link is blocked. The proposed method maximizes the maximum secrecy rate in the information processing stage, and the relays are able to scavenge the energy in both signals of the licensed transmitter and the unlicensed source based on the power splitting protocol. Moreover, the relays must correctly restore the unlicensed source's information before relaying it

(ii) Propose exact closed-form expressions for principal security capability indicators including the SOP, the intercept probability (IP), the probability of strictly positive maximum secrecy rate (PSPMSR) under both licensed outage restriction and peak transmission power restriction, and the licensed interference to quickly assess the secrecy performance of the relay selection in CRNwEH without the need of exhaustive simulations

(iii) Exploit the suggested formulas to optimally select pivotal system parameters

(iv) Supply multiple results to achieve helpful insights into the security capability; for example, the minimum SOP accomplishable with reasonable selection of the time switching ratio, the relays' positions, and the power splitting ratio; the secrecy performance saturation at high peak transmission powers or high preset outage probabilities of licensed users

1.4. Paper Structure. The next part describes maximum secrecy rate, signal model, system model, unlicensed power allocation, and relay selection. Part 3 presents the detailed derivation of essential performance indicators including the IP, the SOP, and the PSPMSR. Part 4 shows analytical/simulated results, and Part 5 closes our work.

\section{System Description}

2.1. System Model. Figure 1 demonstrates the relay selection in $\mathrm{CRNwEH}$. A complete communication from the unlicensed source (US) to the unlicensed destination (UD) with the help of the selected unlicensed relay $\left(\mathrm{UR}_{b}\right)$ comprises two stages as depicted in Figure2(a).

In Stage I, both the licensed transmitter (LT) and US concurrently transmit their legitimate information to the licensed receiver (LR) and UD, correspondingly, inducing mutual interferences between the unlicensed and licensed networks. The unlicensed interferences (i.e., in the unli-

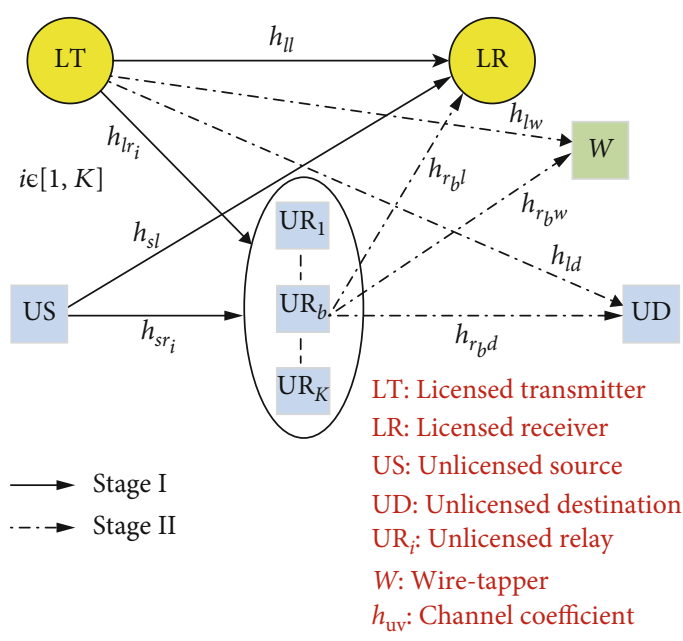

Figure 1: System model.

censed to licensed direction) are well investigated in open literature but the licensed interferences (i.e., in the licensed to unlicensed direction) are normally ignored (e.g., $[5,6,8,17$, $21-26,28,34-37])$. As such, by integrating these mutual interferences into the system model, the current paper is undoubtedly more general than published ones yet the performance analysis is more complicated. The wire-tapper $(W)$ purposely eavesdrops the US's information. Due to bad propagation conditions, the US's signals are unable to be reliably received by UD and $W$. Therefore, it is advisable to ask the unlicensed relays $\left(\mathrm{UR}_{i}\right), i \in[1, K]$, in the transmission range of US for relaying the US's information to UD. In order to save bandwidth and power, this paper just selects one relay, namely, $\mathrm{UR}_{b}$, from a subset of relays which successfully decodes the US's information. Moreover, in order to avoid the waste of energy for relaying operation, the relays are assumed to relay with the energy scavenged from the RF signals. The energy harvesting is implemented with the power splitting protocol (e.g., $[38,39])$ as seen in Figure 2(b). More specifically, the signals of LT and US provide the RF energy for $\mathrm{UR}_{i}$ to harvest. Accordingly, this paper exploits even the interference from LT as a useful energy supply. The power of $\mathrm{UR}_{i}$ 's received signal is split into two portions: one for restoring the US's information (Most previous works (e.g., $[5,12,17-19,21-28,34-39])$ omitted the power consumption of the information decoder. The current paper also follows this assumption) and another for harvesting the energy.

In Stage II, the adopted relay $\mathrm{UR}_{b}$ restores the US's information and forwards the decoded information to UD at the same time that LT transmits its information to LR, which again induces mutual interference between the licensed and unlicensed networks. At the end of Stage II, UD attempts to restore while $W$ wire-taps the US's information from $\mathrm{UR}_{b}$ 's transmit signal.

2.2. Signal Model. In Figure $1, h_{u v} \in\left\{h_{l l}, h_{l w}, h_{l d}, h_{l r_{i}}, h_{s l}, h_{s r_{i}}\right.$, $\left.h_{r_{i}}, h_{r_{i} w}, h_{r_{i} d}\right\}$ symbolizes the channel coefficient between a transmitter-receiver pair (The global channel state information 


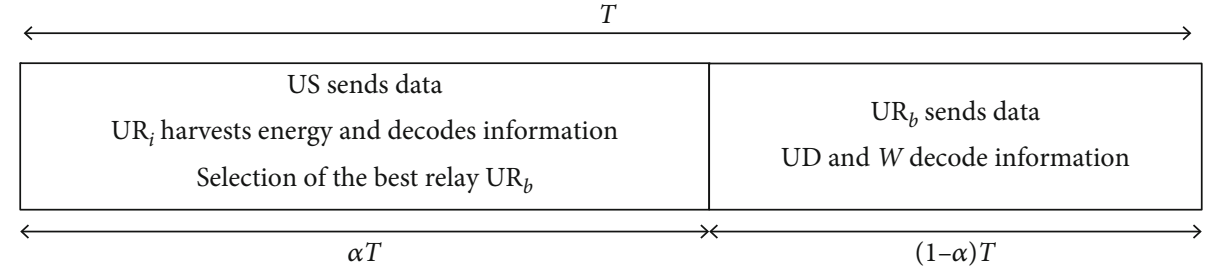

(a) Stage intervals

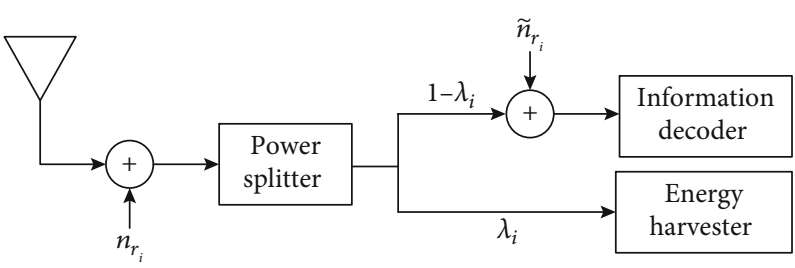

(b) Signal processing at $\mathrm{UR}_{i}$

FIgURE 2: Signal processing.

is assumably available in this paper, which is similar to [6, $8,21]$. How to obtain such global channel state information is beyond our scope but rather we focus on the SOP analysis for the proposed relay selection in $\mathrm{CRNwEH}$ ). Under the assumption of Rayleigh fading, $h_{u v}$ is modelled as a zero-mean $\mu_{u v}$-variance circular symmetric complex Gaussian distribution, i.e., $h_{u v} \sim \mathscr{C} \mathscr{N}\left(0, \mu_{u v}\right)$. Integrating path-loss into channel characteristics, $\mu_{u v}$, is deputized as $\mu_{u v}=\tau_{u v}^{-\omega}$ with $\omega$ symbolizing the path-loss exponent and $\tau_{u v}$ symbolizing the transmitter $u$-receiver $v$ distance. Therefore, it is naturally accepted in the following that the cumulative distribution function $(\mathrm{CDF})$ and the probability density function (PDF) of the channel gain $\left|h_{u v}\right|^{2}$ are correspondingly represented as $F_{\left|h_{u v}\right|^{2}}(x)=1-e^{-x / \mu_{u v}}$ and $f_{\left|h_{u v}\right|^{2}}(x)=e^{-x / \mu_{u v}} / \mu_{u v}$.

In Figure 2, $\alpha$ with $\alpha \in(0,1), \lambda_{i}$ with $\lambda_{i} \in(0,1)$, and $T$ symbolize the time switching ratio, the power splitting ratio, and the total duration for transmission from US to $\mathrm{UD}$ through $\mathrm{UR}_{b}$, respectively. Given these notations in Figure 2, one can model the signals as follows.

$\mathrm{UR}_{i}$ and LR receive signals in Stage I, correspondingly, as

$$
\begin{aligned}
& y_{r_{i}}=h_{s r_{i}} \sqrt{P_{s}} x_{s}+h_{l r_{i}} \sqrt{P_{l}} x_{l}+n_{r_{i}}, \\
& y_{l 1}=h_{s l} \sqrt{P_{s}} x_{s}+h_{l l} \sqrt{P_{l}} x_{l}+n_{l 1},
\end{aligned}
$$

where $x_{s}$ and $x_{l}$ are respectively the unity-power transmit symbols of US and LT; the receive antennas of $\mathrm{UR}_{i}$ and LR generate the noises $n_{r_{i}} \sim \mathscr{C} \mathcal{N}\left(0, \sigma_{r_{i}}^{2}\right)$ and $n_{l 1} \sim \mathscr{C} \mathcal{N}\left(0, \sigma_{l}^{2}\right)$, respectively; the transmission powers of US and LT are $P_{s}$ and $P_{l}$, respectively.

As shown in Figure 2(b), the relay $\mathrm{UR}_{i}$ divides the received signal $y_{r_{i}}$ into two portions: one portion of $\sqrt{\lambda_{i}} y_{r_{i}}$ passes through the energy harvester and another portion of $\sqrt{1-\lambda_{i}} y_{r_{i}}$ passes through the information decoder. Based on $\sqrt{\lambda_{i}} y_{r_{i}}$, the energy that $\mathrm{UR}_{i}$ scavenges in the Stage $\mathrm{I}$ is computed as

$$
E_{r_{i} m}=\eta_{i} \Xi\left\{\left|\sqrt{\lambda_{i}} y_{r_{i}}\right|^{2}\right\} \alpha T=\eta_{i} \lambda_{i}\left(P_{s} \mu_{s r_{i}}+P_{l} \mu_{l r_{i}}+\sigma_{r_{i}}^{2}\right) \alpha T \text {, }
$$

where $\Xi\{\cdot\}$ symbolizes the statistical average and $\eta_{i}$ with $\eta_{i} \in(0,1)$ symbolizes the energy conversion efficiency.

The $\mathrm{UR}_{i}$ consumes the scavenged energy in (3) to transmit information in Stage II with the peak transmission power as

$$
P_{r_{i} m}=\frac{E_{r_{i} m}}{(1-\alpha) T}=\frac{\alpha \eta_{i} \lambda_{i}}{1-\alpha}\left(P_{s} \mu_{s r_{i}}+P_{l} \mu_{l r_{i}}+\sigma_{r_{i}}^{2}\right)
$$

According to the operation principle in Figure 2(b), one can represent the input signal of the information decoder as $\tilde{y}_{r_{i}}=\sqrt{1-\lambda_{i}} y_{r_{i}}+\tilde{n}_{r_{i}}$ where the passband-to-baseband signal converter creates the noise $\tilde{n}_{r_{i}} \sim \mathscr{C} \mathscr{N}\left(0, \tilde{\sigma}_{r_{i}}^{2}\right)$. Inserting (1) in $\tilde{y}_{r_{i}}$ yields $\tilde{y}_{r_{i}}=\sqrt{\left(1-\lambda_{i}\right) P_{s}} h_{s r_{i}} x_{s}+\sqrt{\left(1-\lambda_{i}\right) P_{l}} h_{l r_{i}} x_{l}+$ $\sqrt{1-\lambda_{i}} n_{r_{i}}+\tilde{n}_{r_{i}}$, which creates the SINR (signal-to-interference plus noise ratio) for decoding $x_{s}$ as

$$
\gamma_{s r_{i}}=\frac{\left(1-\lambda_{i}\right) P_{s}\left|h_{s r_{i}}\right|^{2}}{\left(1-\lambda_{i}\right) P_{l}\left|h_{l r_{i}}\right|^{2}+\left(1-\lambda_{i}\right) \sigma_{r_{i}}^{2}+\tilde{\sigma}_{r_{i}}^{2}}=\frac{P_{s}\left|h_{s r_{i}}\right|^{2}}{P_{l}\left|h_{l r_{i}}\right|^{2}+\widehat{\sigma}_{r_{i}}^{2}}
$$

where $\widehat{\sigma}_{r_{i}}^{2}=\sigma_{r_{i}}^{2}+\left(\tilde{\sigma}_{r_{i}}^{2} /\left(1-\lambda_{i}\right)\right)$

Generally, the relay $\mathrm{UR}_{i}$, which is scheduled to transmit in Stage II, will result in the received signals at UD, $W$, and $\mathrm{LR}$, respectively, as 


$$
\begin{gathered}
y_{r_{i} d}=h_{r_{i} d} \sqrt{P_{r_{i}}} x_{r_{i}}+h_{l d} \sqrt{P_{l}} x_{l}+n_{d}, \\
y_{r_{i} w}=h_{r_{i} w} \sqrt{P_{r_{i}}} x_{r_{i}}+h_{l w} \sqrt{P_{l}} x_{l}+n_{w}, \\
y_{r_{i} l}=h_{r_{i} l} \sqrt{P_{r_{i}}} x_{r_{i}}+h_{l l} \sqrt{P_{l}} x_{l}+n_{l 2},
\end{gathered}
$$

where the receive antennas of UD, $W$, and LR create the noises $n_{d} \sim \mathscr{C} \mathcal{N}\left(0, \sigma_{d}^{2}\right), n_{w} \sim \mathscr{C} \mathcal{N}\left(0, \sigma_{w}^{2}\right)$, and $n_{l 2} \sim \mathscr{C} \mathscr{N}\left(0, \sigma_{l}^{2}\right)$, correspondingly; $P_{r_{i}}$ is the transmission power of the relay $\mathrm{UR}_{i}$. It is noted that because LT transmits the signal $x_{l}$ at the same time that $\mathrm{UR}_{i}$ transmits the signal $x_{r_{i}}$, the terms $\left(h_{l d} \sqrt{P_{l}} x_{l}\right.$, $\left.h_{l w} \sqrt{P_{l}} x_{l}, h_{l l} \sqrt{P_{l}} x_{l}\right)$ are the interferences induced by LT to UD and $W$, respectively. Furthermore, in order to decrease the number of notations without loss of generality, equations in (1), (2), (6), (7), and (8) supposed that merely one licensed transmitter-receiver pair are communicating to each other in both stages. That is why the same set of notations $\left(h_{l l}, P_{l}, x_{l}\right)$ is used for both stages in (1), (2), (6), (7), and (8). The case of different licensed transmitter-receiver pairs in two stages is straightforwardly extended.

2.3. Maximum Secrecy Rate. Assuming that the relay $\mathrm{UR}_{i}$ is scheduled to transmit information in Stage II. Then, (6) and (7) yield the SINRs at UD and $W$ as

$$
\begin{gathered}
\gamma_{r_{i} d}=\frac{P_{r_{i}}\left|h_{r_{i} d}\right|^{2}}{P_{l}\left|h_{l d}\right|^{2}+\sigma_{d}^{2}}, \\
\gamma_{r_{i} w}=\frac{P_{r_{i}}\left|h_{r_{i} w}\right|^{2}}{P_{l}\left|h_{l w}\right|^{2}+\sigma_{w}^{2}} .
\end{gathered}
$$

From (9) and (10), channel capacities that UD and $W$ achieve through the assistance of $\mathrm{UR}_{i}$ are respectively given by [40]

$$
\begin{gathered}
C_{r_{i} d}=(1-\alpha) \log _{2}\left(1+\gamma_{r_{i} d}\right), \\
C_{r_{i} w}=(1-\alpha) \log _{2}\left(1+\gamma_{r_{i} w}\right),
\end{gathered}
$$

where the duration of Stage II of $(1-\alpha) T$ indicates the prelog factor of $(1-\alpha)$.

The subtraction of the $\left(\mathrm{UR}_{i}\right.$ to $\left.W\right)$ wire-tap channel capacity from the (UR $\mathrm{UR}_{i}$ to UD) main channel capacity is the maximum secrecy rate [16]. Therefore, with the assistance of $\mathrm{UR}_{i}$, the maximum secrecy rate can be achieved as

$$
C_{r_{i}}=\left[C_{r_{i} d}-C_{r_{i} w}\right]^{+}=(1-\alpha)\left[\log _{2} \frac{1+\gamma_{r_{i} d}}{1+\gamma_{r_{i} w}}\right]^{+},
$$

where $[x]^{+}$denotes $\max (x, 0)$.
2.4. Licensed Power Allocation. From (2), one can compute the SINR at LR in Stage I as

$$
\gamma_{l 1}=\frac{P_{l}\left|h_{l l}\right|^{2}}{P_{s}\left|h_{s l}\right|^{2}+\sigma_{l}^{2}}
$$

from which the channel capacity that LR can obtain in Stage I is

$$
C_{l 1}=\alpha \log _{2}\left(1+\gamma_{l 1}\right)
$$

Similarly, the SINR at LR in Stage II under the activation of the relay $\mathrm{UR}_{i}$ is inferred from (8) as

$$
\gamma_{l 2 i}=\frac{P_{l}\left|h_{l l}\right|^{2}}{P_{r_{i}}\left|h_{r_{i} l}\right|^{2}+\sigma_{l}^{2}}
$$

from which the channel capacity that LR can obtain in Stage II under the activation of the relay $\mathrm{UR}_{i}$ is

$$
C_{l 2 i}=(1-\alpha) \log _{2}\left(1+\gamma_{l 2 i}\right)
$$

In cognitive radio networks, the reliability of the licensed users is of paramount importance. Therefore, the unlicensed transmitters (US and $\mathrm{UR}_{i}$ ) must guarantee the reliability of the licensed users while they operate. In this paper, the reliability of the licensed users is represented by the outage probability of the licensed receiver. As such, the transmission powers of the unlicensed users must be controlled for the outage probability of the licensed receiver not to exceed a preset value $\varepsilon$. To be specific, $P_{s}$ and $P_{r_{i}}$ must be subject to

$$
\operatorname{Pr}\left\{C_{l 1} \leq C_{2}\right\} \leq \varepsilon
$$

$$
\operatorname{Pr}\left\{C_{l 2 i} \leq C_{2}\right\} \leq \varepsilon,
$$

where $C_{2}$ is the target spectral efficiency of LR.

Restrictions in (17) and (18) are named as the licensed outage restrictions.

The transmission powers of US and $\mathrm{UR}_{i}$ are also upperbounded by their peak transmission powers, $P_{s m}$ and $P_{r_{i} m}$, correspondingly, that are due to the hardware design and the energy harvester, respectively. As such, $P_{s}$ and $P_{r_{i}}$ are constrained by

$$
\begin{aligned}
& P_{s} \leq P_{s m}, \\
& P_{r_{i}} \leq P_{r_{i} m} .
\end{aligned}
$$

Restrictions in (19) and (20) are named as the peak transmission power restrictions. 
The solution of the equation system (i.e., the qualities hold for (17) and (19)) is the transmission power of $P_{s}$ as

$$
P_{s}=\min \left(\frac{P_{l} \mu_{l l}}{\gamma_{21} \mu_{s l}}\left[\frac{1}{1-\varepsilon} e^{-\gamma_{21} \sigma_{l}^{2} / P_{l} \mu_{l l}}-1\right]^{+}, P_{s m}\right)
$$

where $\gamma_{21}=2^{C_{2} / \alpha}-1$

Similarly, the solution of the equation system (i.e., the qualities hold for (18) and (20)) is the transmission power of $P_{r_{i}}$ as

$$
P_{r_{i}}=\min \left(\frac{P_{l} \mu_{l l}}{\gamma_{22} \mu_{r_{i} l}}\left[\frac{1}{1-\varepsilon} e^{-\gamma_{22} \sigma_{l}^{2} / P_{l} \mu_{l l}}-1\right]^{+}, P_{r_{i} m}\right)
$$

where $\gamma_{22}=2^{C_{2} /(1-\alpha)}-1$.

The equations in (21) and (22) are derived similarly as ([3], eq. (17)) and ([3], eq. (19)).

2.5. Relay Selection. According to information theory, $\mathrm{UR}_{i}$ can achieve the channel capacity in Stage $\mathrm{I}$ as $C_{s r_{i}}=\alpha \log _{2}\left(1+\gamma_{s r_{i}}\right) \quad \mathrm{bps} / \mathrm{Hz}$ where the reason for the appearance of the prelog factor $\alpha$ is that the duration of Stage $\mathrm{I}$ is $\alpha T$. Moreover, according to information theory, $\mathrm{UR}_{i}$ can recover the US's information as long as $C_{s r_{i}}$ exceeds the target spectral efficiency of SUs, $C_{1}$, i.e., $C_{s r_{i}} \geq C_{1}$. Equivalently, $x_{s}$ is correctly restored at $\mathrm{UR}_{i}$ if $\gamma_{s r_{i}} \geq \gamma_{1}$ where $\gamma_{1}=2^{C_{1} / \alpha}-1$.

Let $\mathscr{D}$ be the set of relays that correctly restored the US's message, i.e.,

$$
\mathscr{D}=\left\{U R_{i}: C_{s r_{i}} \geq C_{1}\right\} \text {. }
$$

Among relays in $\mathscr{D}$, the relay, namely, $\mathrm{UR}_{b}$, that maximizes the maximum secrecy rate is chosen to relay the US's information in Stage II. According to this relay selection method, the maximum secrecy rate that $\mathrm{CRNwEH}$ can achieve is given by

$$
C_{\mathrm{sec}}=\max _{r_{i} \in \mathscr{D}} C_{r_{i}}=\max _{r_{i} \in \mathscr{D}}(1-\alpha)\left[\log _{2}\left(\frac{1+\gamma_{r_{i}} d}{1+\gamma_{r_{i}} w}\right)\right]^{+},
$$

which is essential to derive the performance metric for the proposed relay selection method in $\mathrm{CRNwEH}$.

\section{SOP Analysis}

In the viewpoint of information theory, the most appropriate performance metric for evaluating the security capability of wireless transmission is the SOP, which is the possibility that $C_{\text {sec }}$ is below a target security degree $\bar{C}_{3}$. Therefore, the lower SOP indicates the more secure wireless transmission. This part derives elaborately the SOP for the suggested relay selection method in $\mathrm{CRNwEH}$. The derived closed-form expression of the SOP facilitates in evaluating the secrecy performance without time-consuming simulations and in deriving other paramount security capability indicators, e.g., the PSPMSR and the IP.

The SOP of the relay selection in $\mathrm{CRNwEH}$ is expressed as

$\mathcal{U}\left(\bar{C}_{3}\right)=\operatorname{Pr}\left\{C_{\mathrm{sec}}<\bar{C}_{3}\right\}=\operatorname{Pr}\left\{\max _{r_{i} \in \mathscr{D}}(1-\alpha)\left[\log _{2}\left(\frac{1+\gamma_{r_{i} d}}{1+\gamma_{r_{i}} w}\right)\right]^{+}<\bar{C}_{3}\right\}$.

Because the number of relays which restore correctly the US's message in Stage I varies from 0 to $K$, according to the total probability principle, (25) can be decomposed as

$$
\begin{aligned}
\mathscr{U}\left(\bar{C}_{3}\right)= & \operatorname{Pr}\left\{0<\bar{C}_{3}|| \mathscr{D} \mid=0\right\} \operatorname{Pr}\{|\mathscr{D}|=0\}+\sum_{k=1}^{K} \operatorname{Pr} \\
& \cdot\left\{\max _{r_{i} \in \mathscr{D}}(1-\alpha)\left[\log _{2}\left(\frac{1+\gamma_{r_{i}} d}{1+\gamma_{r_{i}}}\right)\right]^{+}<\bar{C}_{3}|| \mathscr{D} \mid=k\right\} \operatorname{Pr}\{|\mathscr{D}|=k\},
\end{aligned}
$$

where $|\cdot|$ symbolizes the cardinality of the set.

The target security degree $\bar{C}_{3}$ is nonnegative and hence, (26) can be simplified as

$$
\mathscr{U}\left(\bar{C}_{3}\right)=\underbrace{\operatorname{Pr}\{|\mathscr{D}|=0\}^{\prime}}_{Q_{1}}+\sum_{k=1}^{K} \sum_{m=1}^{\left(\begin{array}{c}
K \\
k
\end{array}\right)} \underbrace{\operatorname{Pr}\left\{\max (1-\alpha)\left[\log _{2}\left(\frac{1+\gamma_{r_{i} d}}{1+\gamma_{r_{i} w}}\right)\right]^{+}<\bar{C}_{3}\right\}}_{Q_{2}} \underbrace{\operatorname{Pr}\left\{\delta_{m}^{k}\right\}}_{Q_{3}} .
$$


In (27), $\left(\begin{array}{l}K \\ k\end{array}\right)=K ! / k !(K-k)$ ! denotes the binomial coefficient and $\delta_{m}^{k}$ stands for the $m^{\text {th }}$ combination among $\left(\begin{array}{l}K \\ k\end{array}\right)$ combinations, each comprising $k$ distinct elements taken from the set of $K$ different elements. For instance, $\delta_{1}^{2}=\{1,2\}, \delta_{2}^{2}=\{1,3\}, \delta_{3}^{2}=\{2,3\}$ for $k=2$ and $K=3$.

In order to represent (27) in closed-form, the triplet $\left(\mathbb{Q}_{1}\right.$, $Q_{2}, Q_{3}$ ) will be solved sequentially as follows.

The quantity $\mathbb{Q}_{1}$ is the possibility which all relays fail to restore the US's information in Stage I. As such, $\mathbb{Q}_{1}$ can be computed as

$$
Q_{1}=\operatorname{Pr}\left\{\bigcap_{i=1}^{K}\left\{\gamma_{s r_{i}}<\gamma_{1}\right\}\right\} .
$$

Because of the statistical independence of $\gamma_{s r_{i}}, i=\{1, \cdots$, $K$ \}, one can rewrite (28) as

$$
Q_{1}=\prod_{i=1}^{K} \underbrace{\operatorname{Pr}\left\{\gamma_{s r_{i}}<\gamma_{1}\right\}}_{\mathscr{F}_{i}},
$$

where $\mathscr{I}_{i}$ has the precise closed form as

$$
\mathscr{I}_{i}=1-O_{i} \frac{e^{-G_{i} \gamma_{1}}}{\gamma_{1}+O_{i}}
$$

with

$$
\begin{gathered}
O_{i}=\frac{\mu_{s r_{i}} P_{s}}{\mu_{l r_{i}} P_{l}}, \\
G_{i}=\frac{\widehat{\sigma}_{r_{i}}^{2}}{\mu_{s r_{i}} P_{s}} .
\end{gathered}
$$

The proof of (30) is presented in Appendix A.

The quantity $\mathbb{Q}_{3}$ is the probability that the relays belonging to the set $\delta_{m}^{k}$ successfully decode the US's information while the relays not belonging to the set $\delta_{m}^{k}$ unsuccessfully decode the US's information. Therefore, $\mathbb{Q}_{3}$ is represented in closed-form as

$$
Q_{3}=\operatorname{Pr}\left\{\bigcap_{i \in \mathcal{S}_{m}^{k}}\left\{\gamma_{s r_{i}} \geq \gamma_{1}\right\}, \bigcap_{j \notin \mathcal{S}_{m}^{k}}\left\{\gamma_{s r_{j}}<\gamma_{1}\right\}\right\} .
$$

Because of the statistical independence of $\gamma_{s r_{i}}, i=\{1, \cdots$, $K\}$, one can rewrite (33) as

$$
\mathbb{Q}_{3}=\prod_{i \in \mathcal{S}_{m}^{k}}\left(1-\mathscr{I}_{i}\right) \prod_{j \notin \mathcal{S}_{m}^{k}} \mathscr{I}_{j}
$$

where $\mathscr{I}_{i}$ is given by (30) while $\mathscr{I}_{j}$ is also given by (30) with $i$ being replaced by $j$.

The last quantity $Q_{2}$ is decomposed as

$$
Q_{2}=\operatorname{Pr}\left\{\bigcap_{r_{i} \in \delta_{m}^{k}}\left\{\left[\log _{2}\left(\frac{1+\gamma_{r_{i} d}}{1+\gamma_{r_{i}} w}\right)\right]^{+}<\frac{\bar{C}_{3}}{1-\alpha}\right\}\right\} .
$$

Terms $\left(1+\gamma_{r_{i} d}\right) /\left(1+\gamma_{r_{i} w}\right)$ are statistically dependent because they contain two common variables $\left(\left|h_{l d}\right|^{2}\right.$ and $\left.\left|h_{l w}\right|^{2}\right)$. To decorrelate them, one applies the conditional probability as

$Q_{2}=\Xi_{\left|h_{l d}\right|^{2},\left|h_{w w}\right|^{2}}\left\{\prod_{r_{i} \in S_{m}^{k}}^{\operatorname{Pr}\left\{\left[\log _{2}\left(\frac{1+\gamma_{r_{i} d}}{1+\gamma_{r_{i} w}}\right)\right]^{+}<\left.C_{3}|| h_{l d}\right|^{2},\left|h_{l w}\right|^{2}\right\}}\right\}$,

where

$$
C_{3}=\frac{\bar{C}_{3}}{1-\alpha} .
$$

The term $\mathscr{G}_{i}$ is represented in closed-form as

$$
\mathscr{G}_{i}=1-\frac{\left(C_{i}\left|h_{l w}\right|^{2}+D_{i}\right) e^{-\left(2^{C_{3}-1}\right)\left(A_{i}\left|h_{l d}\right|^{2}+B_{i}\right)}}{2^{C_{3}}\left(A_{i}\left|h_{l d}\right|^{2}+B_{i}\right)+C_{i}\left|h_{l w}\right|^{2}+D_{i}},
$$

where

$$
\begin{aligned}
& A_{i}=\frac{P_{l}}{\mu_{r_{i} d} P_{r_{i}}}, \\
& B_{i}=\frac{\sigma_{d}^{2}}{\mu_{r_{i} d} P_{r_{i}}}, \\
& C_{i}=\frac{P_{l}}{\mu_{r_{i} w} P_{r_{i}}}, \\
& D_{i}=\frac{\sigma_{w}^{2}}{\mu_{r_{i} w} P_{r_{i}}} .
\end{aligned}
$$

The proof of (38) is presented in Appendix B. For simplicity, one lets

$$
\begin{aligned}
& X=\left|h_{l d}\right|^{2}, \\
& Y=\left|h_{l w}\right|^{2}, \\
& a_{i}=\frac{\left(C_{i} Y+D_{i}\right) e^{-\left(2^{C_{3}-1}\right)\left(A_{i} X+B_{i}\right)}}{2^{C_{3}}\left(A_{i} X+B_{i}\right)+C_{i} Y+D_{i}} .
\end{aligned}
$$

Then, $\mathscr{G}_{i}$ in (38) has a compact form as

$$
\mathscr{G}_{i}=1-a_{i} .
$$


Inserting (46) into (36), one has

$$
Q_{2}=\Xi_{X, Y}\left\{\prod_{r_{i} \in \mathcal{S}_{m}^{k}}\left(1-a_{i}\right)\right\} .
$$

Using the following equality ([3], eq. (30))

$$
\begin{aligned}
\prod_{k=1}^{L}\left(1-a_{k}\right)= & +\sum_{u=1}^{L-1}(-1)^{u} \sum_{v_{1}=1}^{L-u+1} \sum_{v_{2}=v_{1}+1}^{L-u+2} \cdots \sum_{v_{u}=v_{u-1}+1}^{L} \prod_{k \in \mathscr{M}} a_{k} \\
& +(-1)^{L} \prod_{k \in \mathscr{R}} a_{k},
\end{aligned}
$$

with $\mathscr{R}=\{1,2, \cdots, L\} \quad$ and $\mathscr{M}=\left\{\mathscr{R}\left[v_{1}\right], \cdots, \mathscr{R}\left[v_{u}\right]\right\} \quad$ to expand (48) as

$$
\begin{aligned}
\mathscr{Q}_{2}= & +\sum_{u=1}^{\left|\delta_{m}^{k}\right|-1}(-1)^{u} \sum_{v_{1}=1}^{\left|\delta_{m}^{k}\right|-u+1} \sum_{v_{2}=v_{1}+1}^{\left|\delta_{m}^{k}\right|-u+2} \cdots \sum_{v_{u}=v_{u-1}+1}^{\left|\delta_{m}^{k}\right|} \underbrace{\Xi_{X, Y}\left\{\prod_{i \in \mathscr{A}} a_{i}\right\}}_{Q_{2, \mathscr{A}}} \\
& +(-1) \mid \underbrace{\left|\delta_{m}^{k}\right|}_{\mathcal{Q}_{2 \delta_{m}^{k}}} \underbrace{}_{\Xi_{X, Y}\left\{\prod_{i \in \delta_{m}^{k}} a_{i}\right\}},
\end{aligned}
$$

where $\mathscr{A}=\left\{\mathcal{S}_{m}^{k}\left[v_{1}\right], \cdots, \mathcal{S}_{m}^{k}\left[v_{u}\right]\right\}$.

To obtain the closed form of $Q_{2}$, one needs to evaluate a common expression

$$
\mathcal{Q}_{2 \mathcal{W}}=\Xi_{X, Y}\left\{\prod_{i \in \mathscr{W}} a_{i}\right\}
$$

where $\mathscr{W}=\left\{\mathscr{A}, \mathcal{S}_{m}^{k}\right\}$.

Inserting (45) into (50), one obtains

$$
\begin{aligned}
& Q_{2 \mathscr{W}}=\Xi_{X, Y}\left\{\prod_{i \in \mathscr{W}} \frac{\left(C_{i} Y+D_{i}\right) e^{-\left(2^{C_{3}}-1\right)\left(A_{i} X+B_{i}\right)}}{2^{C_{3}} A_{i} X+C_{i} Y+2^{C_{3}} B_{i}+D_{i}}\right\} \\
& =e^{-\left(2^{C_{3}}-1\right) \sum_{i \in \mathscr{W}} B_{i}}\left(\prod_{i \in \mathscr{W}} \frac{1}{2^{C_{3}} A_{i}}\right) \Xi_{X, Y}\left\{e^{-X\left(2^{C_{3}}-1\right) \sum_{i \in \mathscr{W}} \prod_{i}} \frac{C_{i} Y+D_{i}}{X+2^{-C_{3}}\left(C_{i} / A_{i}\right) Y+\left(B_{i}+2^{-C_{3}} D_{i}\right) / A_{i}}\right\} \\
& =e^{-\left(2^{C_{3}}-1\right) \sum_{i \in \mathscr{W}} B_{i}}\left(\prod_{i \in \mathscr{W}} \frac{1}{2^{C_{3}} A_{i}}\right) \times \underbrace{\Xi_{Y}\{\left[\prod_{i \in \mathscr{W}}\left(C_{i} Y+D_{i}\right)\right] \underbrace{\Xi_{X}\left\{e^{-X\left(2^{C_{3}}-1\right) \sum_{i \in \mathscr{W}} A_{i}} \prod_{i \in \mathscr{W}}\left[X+2^{-C_{3}} \frac{C_{i}}{A_{i}} Y+\frac{B_{i}+2^{-C_{3} D_{i}}}{A_{i}}\right]\right\}}_{\mathscr{K}}\}}_{\mathscr{H}} .
\end{aligned}
$$

The term $\mathscr{K}$ can be rewritten in an explicit form as

$$
\begin{aligned}
& \mathscr{K}=\int_{0}^{\infty} \frac{1}{\mu_{l d}} e^{-x / \mu_{l d}} e^{-x\left(2^{C_{3}}-1\right) \sum_{i \in \mathscr{W}} A_{i}} \prod_{i \in \mathscr{W}}\left[x+2^{-C_{3}} \frac{C_{i}}{A_{i}} Y+\frac{B_{i}+2^{-C_{3}} D_{i}}{A_{i}}\right]^{-1} d x \\
& =\frac{1}{\mu_{l d}} \int_{0}^{\infty} e^{-x\left[1 / \mu_{l d}+\left(2^{C_{3}}-1\right) \sum_{i \in \mathscr{W}} A_{i}\right]} \sum_{i \in \mathscr{W}} H_{i} /\left(x+2^{-C_{3}} \frac{C_{i}}{A_{i}} Y+\frac{B_{i}+2^{-C_{3}} D_{i}}{A_{i}}\right) d x,
\end{aligned}
$$

where

$$
H_{i}=\prod_{\substack{j \in \mathscr{W} \\ j \neq i}}\left[2^{-C_{3}}\left(\frac{C_{j}}{A_{j}}-\frac{C_{i}}{A_{i}}\right) Y+\frac{B_{j}+2^{-C_{3}} D_{j}}{A_{j}}-\frac{B_{\mathrm{i}}+2^{-C_{3}} D_{i}}{A_{i}}\right]^{-1},
$$

By exchanging the summation and the integration, one obtains

$$
\begin{aligned}
& \mathscr{K}=\frac{1}{\mu_{l d}} \sum_{i \in \mathscr{W}} H_{i} \int_{0}^{\infty} \frac{e^{-\left[1 / \mu_{l d}+\left(2^{C_{3}}-1\right) \sum_{i \in \mathscr{W}} A_{i}\right] x}}{x+2^{-C_{3}}\left(C_{i} / A_{i}\right) Y+\left(B_{i}+2^{-C_{3}} D_{i}\right) / A_{i}} d x \\
& \left.=-\frac{1}{\mu_{l d}} \sum_{i \in \mathscr{W}} H_{i} e^{\left[1 / \mu_{l d}+\left(2^{C_{3}}-1\right)\right.} \sum_{i \in \mathscr{W}} A_{i}\right]\left(2^{\left.-C_{3} \frac{C_{i}}{A_{i}} Y+\frac{B_{i}+2^{-C} C_{3 D_{i}}}{A_{i}}\right)}\right. \\
& \times E i\left(-\left[\frac{1}{\mu_{l d}}+\left(2^{C_{3}}-1\right) \sum_{i \in \mathscr{W}} A_{i}\right]\left(2^{-C_{3}} \frac{C_{i}}{A_{i}} Y+\frac{B_{i}+2^{-C_{3}} D_{i}}{A_{i}}\right)\right),
\end{aligned}
$$

where $\operatorname{Ei}(\cdot)$ is the exponential integral in [41], and the 
integral in the first equality is achieved from the following result:

$$
\Omega(a, b)=\int_{0}^{\infty} \frac{e^{-a x}}{x+b} d x=-e^{a b} E i(-a b) .
$$

Inserting $H_{i}$ in (53) into (54) and performing simplifications, one reduces $\mathscr{K}$ to

$$
\mathscr{K}=-\frac{1}{\mu_{l d}} \sum_{i \in \mathscr{W}} L_{i}\left(\prod_{\substack{j \in \mathscr{W} \\ j \neq i}} \frac{1}{Y+M_{j}}\right) e^{V_{i} Y} \operatorname{Ei}\left(-V_{i} Y-U_{i}\right),
$$

where $\quad U_{i}=\left[1 / \mu_{l d}+\left(2^{C_{3}}-1\right) \sum_{i \in \mathscr{W}} A_{i}\right]\left(B_{i}+2^{-C_{3}} D_{i}\right) / A_{i}, \quad V_{i}$ $=\left[1 / \mu_{l d}+\left(2^{C_{3}}-1\right) \sum_{i \in \mathscr{W}} A_{i}\right] 2^{-C_{3}} C_{i} / A_{i}, \quad M_{j}=\left(\left(B_{j}+2^{-C_{3}} D_{j}\right) /\right.$ $\left.A_{j}-\left(B_{i}+2^{-C_{3}} D_{i}\right) / A_{i}\right) 2^{C_{3}}\left(C_{j} / A_{j}-C_{i} / A_{i}\right)^{-1}, \quad$ and $\quad L_{i}=e^{U_{i}} /$ $\prod_{j \in \mathscr{W}} 2_{j \neq i}^{-C_{3}}\left(C_{j} / A_{j}-C_{i} / A_{i}\right)$.

The term $\mathscr{H}$ in (51) can be written in an explicit form as

$$
\begin{aligned}
\mathscr{H}= & \int_{0}^{\infty} \frac{1}{\mu_{l w}} e^{-y / \mu_{l w}}\left\{\prod_{i \in \mathscr{W}}\left(C_{i} y+D_{i}\right)\right\} \mathscr{K} d y \\
= & -\int_{0}^{\infty} \frac{1}{\mu_{l w}} e^{-y / \mu_{l w}}\left\{\prod_{i \in \mathscr{W}}\left(C_{i} y+D_{i}\right)\right\} \frac{1}{\mu_{l d}} \sum_{i \in \mathscr{W}} L_{i} \\
& \cdot\left\{\prod_{j \in \mathscr{W}} \frac{1}{y+i}\right\} e^{V_{i} y} E i\left(-V_{i} y-U_{i}\right) d y \\
= & -\frac{\prod_{i \in \mathscr{W}} C_{i}}{\mu_{l w} \mu_{l d}} \sum_{i \in \mathscr{W}} L_{i} \int_{0}^{\infty} e^{-\left(1 / \mu_{l w}-V_{i}\right) y}\left(y+\frac{D_{i}}{C_{i}}\right) \\
& \cdot\left\{\prod_{j \in \mathscr{W}} \frac{y+D_{j} / C_{j}}{y+M_{j}}\right\} E i\left(-V_{i} y-U_{i}\right) d y .
\end{aligned}
$$

By denoting $\mathcal{X}=\mathcal{W} \backslash i$, one can rewrite $\mathscr{H}$ as

$$
\begin{aligned}
\mathscr{H}= & -\frac{\prod_{i \in \mathscr{W}} C_{i}}{\mu_{l w} \mu_{l d}} \sum_{i \in \mathscr{W}} L_{i} \int_{0}^{\infty} e^{-\left(1 / \mu_{l w}-V_{i}\right) y}\left(y+\frac{D_{i}}{C_{i}}\right) \\
& \cdot\left\{\prod_{j \in \mathscr{X}}\left(1-\frac{M_{j}-D_{j} / C_{j}}{y+M_{j}}\right)\right\} E i\left(-V_{i} y-U_{i}\right) d y .
\end{aligned}
$$

Using (48) to decompose $\mathscr{H}$ as

$$
\begin{aligned}
\mathscr{H}= & -\frac{\prod_{i \in \mathscr{W}} C_{i}}{\mu_{l w} \mu_{l d}} \sum_{i \in \mathscr{V}} L_{i} \int_{0}^{\infty} e^{-\left(1 / \mu_{l w}-V_{i}\right) y}\left(y+\frac{D_{i}}{C_{i}}\right) E i\left(-V_{i} y-U_{i}\right) \\
& \times\left\{1+\sum_{q=1}^{|X|-1}(-1)^{q} \sum_{u_{1}=1}^{|X|-q+1|X|-q+2} \sum_{u_{2}=u_{1}+1} \cdots \sum_{u_{q}=u_{q-1}+1}^{|X|} \prod_{j \in \mathcal{Z}} b_{j}+(-1)^{|X|} \prod_{k \in \mathcal{X}} b_{k}\right\} d y,
\end{aligned}
$$

where $\mathscr{Z}=\left\{\mathscr{X}\left[u_{1}\right], \cdots, \mathscr{X}\left[u_{q}\right]\right\}$ and

$$
b_{j}=\frac{M_{j}-D_{j} / C_{j}}{y+M_{j}} .
$$

One can further simplify (59) as

$$
\begin{aligned}
\mathscr{H}= & -\frac{\prod_{i \in \mathscr{W}} C_{i}}{\mu_{l w} \mu_{l d}} \sum_{i \in \mathscr{W}} L_{i} \\
& \cdot\left\{\Phi_{\varnothing}+\sum_{q=1}^{|\mathscr{X}|-1}(-1)^{q} \sum_{u_{1}=1}^{|X|-q+1} \sum_{u_{2}=u_{1}+1}^{|X|-q+2} \ldots \sum_{u_{q}=u_{q-1}+1}^{|X|} \Phi_{\mathcal{Z}}+(-1)^{|X|} \Phi_{\mathscr{X}}\right\},
\end{aligned}
$$

where

$$
\begin{aligned}
\Phi_{\varnothing} & =\int_{0}^{\infty} e^{-\left(1 / \mu_{l w}-V_{i}\right) y}\left(y+\frac{D_{i}}{C_{i}}\right) \operatorname{Ei}\left(-V_{i} y-U_{i}\right) d y, \\
\Phi_{\mathscr{B}} & =\int_{0}^{\infty} e^{-\left(1 / \mu_{l w}-V_{i}\right) y}\left(y+\frac{D_{i}}{C_{i}}\right) \operatorname{Ei}\left(-V_{i} y-U_{i}\right)\left\{\prod_{j \in \mathscr{B}} b_{j}\right\} d y,
\end{aligned}
$$

with $\mathscr{B}=\{\mathscr{Z}, \mathscr{X}\}$.

The following solves $\Phi_{\varnothing}$ and $\Phi_{\mathscr{B}}$ in closed forms to finish the computation of $\mathscr{H}$. First of all, $\Phi_{\varnothing}$ can be represented in terms of two special functions as

$$
\Phi_{\varnothing}=\Psi\left(\frac{1}{\mu_{l w}}-V_{i}, V_{i}, U_{i}\right)+\frac{D_{i}}{C_{i}} \Theta\left(\frac{1}{\mu_{l w}}-V_{i}, V_{i}, U_{i}\right),
$$

where $\Psi(b, c, d)$ and $\Theta(b, c, d)$ are respectively defined as

$$
\begin{aligned}
& \Psi(b, c, d)=\int_{0}^{\infty} x e^{-b x} E i(-c x-d) d x \\
& \Theta(b, c, d)=\int_{0}^{\infty} e^{-b x} E i(-c x-d) d x
\end{aligned}
$$

Applying ([42], eq. (16)) with appropriate substitutions yields the closed form of $\Theta(b, c, d)$ as

$$
\Theta(b, c, d)=\frac{E i(-d)}{b}+\frac{e^{-d}}{b} \Omega\left(b+c, \frac{d}{c}\right),
$$

where $\Omega(\cdot, \cdot)$ is the function defined in (55). 
TABle 1: Key specifications.

\begin{tabular}{lc}
\hline Specification & Value \\
\hline Coordinate of $\mathrm{LR}$ & $\mathrm{LR}$ at $(0.7,0.6)$ \\
Coordinate of $\mathrm{LT}$ & $\mathrm{LT}$ at $(0.2,0.8)$ \\
Coordinate of $\mathrm{UD}$ & $\mathrm{UD}$ at $(1.0,0.0)$ \\
Coordinate of $\mathrm{UR}_{1}$ & $\mathrm{UR}_{1}$ at $(0.3402,0.0421)$ \\
Coordinate of $\mathrm{UR}_{2}$ & $\mathrm{UR}_{2}$ at $(0.2063,0.1785)$ \\
Coordinate of $\mathrm{UR}_{3}$ & $\mathrm{UR}_{3}$ at $(0.3132,0.1696)$ \\
Coordinate of $\mathrm{UR}_{4}$ & $\mathrm{UR}_{4}$ at $(0.3954,-0.1489)$ \\
Coordinate of $\mathrm{UR}_{5}$ & $\mathrm{UR}_{5}$ at $(0.2534,0.0243)$ \\
Coordinate of $\mathrm{US}$ & $\mathrm{US}$ at $(0.0,0.0)$ \\
Coordinate of $W$ & $W$ at $(1.0,0.4)$ \\
Energy conversion efficiency & $\eta=0.9$ \\
Path-loss exponent & $\omega=4$ \\
\hline
\end{tabular}

Similarly, applying ([43], eq. (29)) with appropriate substitutions yields the closed form of $\Psi(b, c, d)$ as

$\Psi(b, c, d)=\frac{E i(-d)}{b^{2}}+\frac{e^{-d}}{b(b+c)}+\frac{e^{-d}}{b}\left(\frac{1}{b}-\frac{d}{c}\right) \Omega\left(b+c, \frac{d}{c}\right)$.

Inserting (60) into (63), one obtains

$\Phi_{\mathscr{B}}=\int_{0}^{\infty} e^{-\left(1 / \mu_{l w}-V_{i}\right) y}\left(y+\frac{D_{i}}{C_{i}}\right) E i\left(-V_{i} y-U_{i}\right) \prod_{j \in \mathscr{B}} \frac{M_{j}-D_{j} / C_{j}}{y+M_{j}} d y$.

Performing the partial fraction decomposition, one simplifies (68) as

$$
\begin{aligned}
\Phi_{\mathscr{B}}= & \left\{\prod_{j \in \mathscr{B}}\left(M_{j}-\frac{D_{j}}{C_{j}}\right)\right\} \int_{0}^{\infty} e^{-\left(1 / \mu_{l w}-V_{i}\right) y} \\
& \cdot\left(y+\frac{D_{i}}{C_{i}}\right) E i\left(-V_{i} y-U_{i}\right)\left(\sum_{j \in \mathscr{B}} \frac{Q_{j}}{y+M_{j}}\right) d y,
\end{aligned}
$$

where $Q_{j}=\prod_{g \in \mathscr{B}, g \neq j} 1 /\left(M_{g}-M_{j}\right)$.

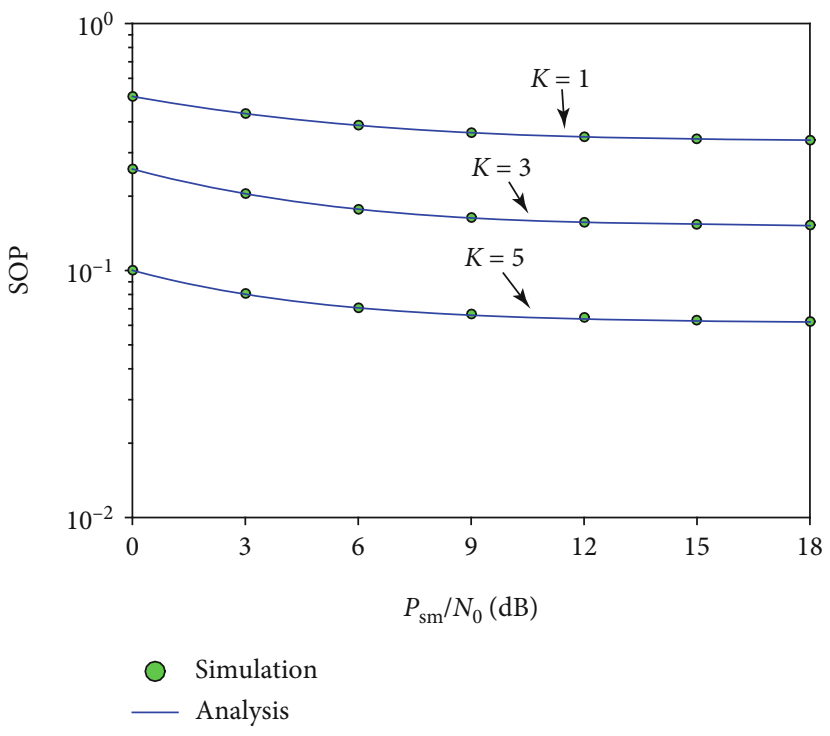

Figure 3: SOP versus $P_{s m} / N_{0}$.

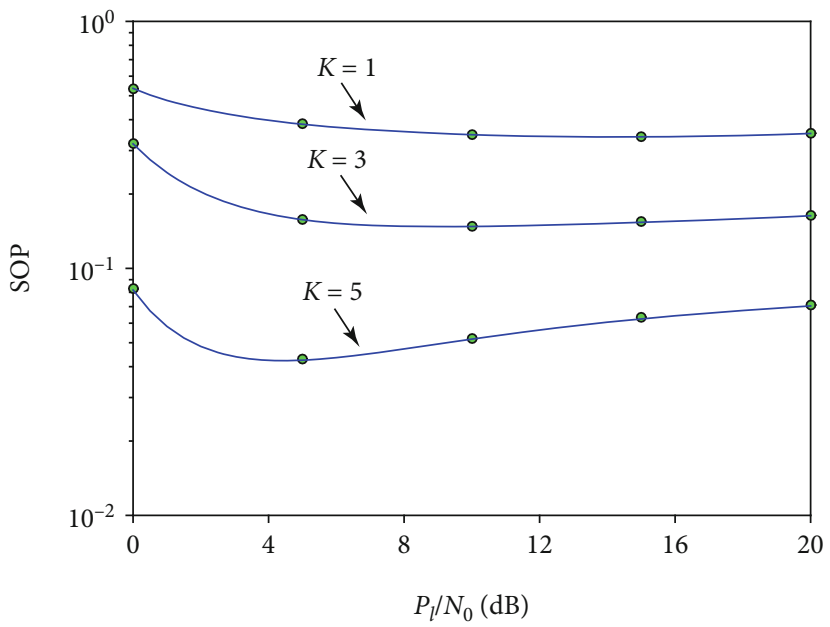

Simulation

— Analysis

Figure 4: SOP versus $P_{l} / N_{0}$.

By exchanging the summation and the integration, (69) is reduced to

$$
\begin{aligned}
\Phi_{\mathscr{B}} & =\left\{\prod_{j \in \mathscr{B}}\left(M_{j}-\frac{D_{j}}{C_{j}}\right)\right\} \sum_{j \in \mathscr{B}} Q_{j} \int_{0}^{\infty} e^{-\left(1 / \mu_{l w}-V_{i}\right) y} \frac{y+D_{i} / C_{i}}{y+M_{j}} E i\left(-V_{i} y-U_{i}\right) d y \\
& =\left\{\prod_{j \in \mathscr{B}}\left(M_{j}-\frac{D_{j}}{C_{j}}\right)\right\} \sum_{j \in \mathscr{B}} Q_{j}\{\underbrace{\int_{0}^{\infty} e^{-\left(1 / \mu_{l w}-V_{i}\right) y} E i\left(-V_{i} y-U_{i}\right) d y}_{\Theta\left(\frac{1}{\mu_{l w}}-V_{i}, V_{i}, U_{i}\right)}+\left(\frac{D_{i}}{C_{i}}-M_{j}\right) \underbrace{\left.\int_{0}^{\infty} \frac{e^{-\left(1 / \mu_{l w}-V_{i}\right) y}}{y+M_{j}} E i\left(-V_{i} y-U_{i}\right) d y\right\}}_{\Lambda\left(\frac{1}{\mu_{w}}-V_{i}, M_{j}, V_{i}, U_{i}\right)},
\end{aligned}
$$



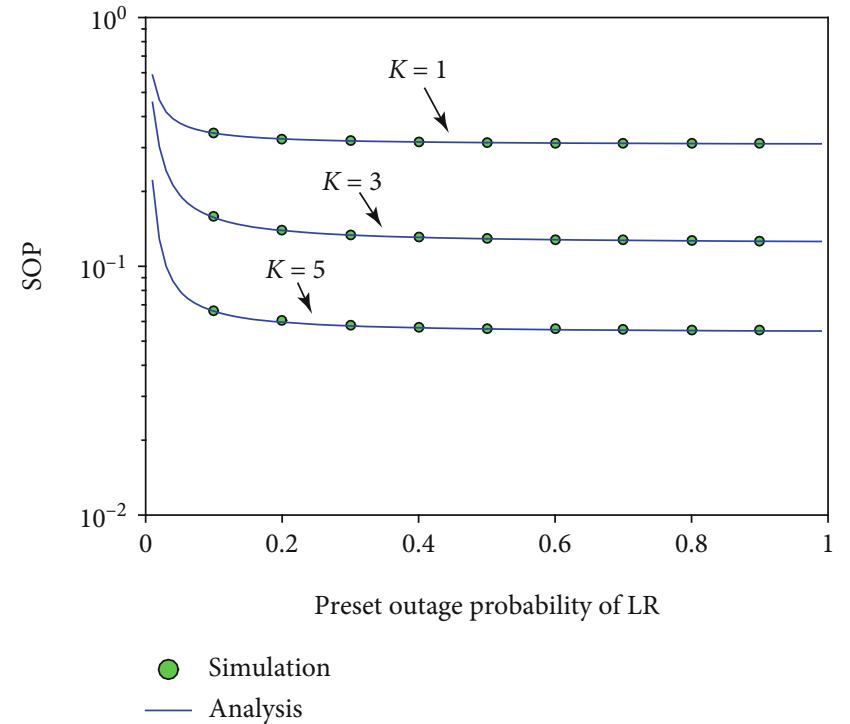

FIGURE 5: SOP versus the preset outage probability of licensed user. where

$$
\Lambda(b, c, g, l)=\int_{0}^{\infty} \frac{e^{-b x}}{x+c} E i(-g x-l) d x
$$

The function $\Lambda(b, c, g, l)$ has an accurate closed form as ([42], eq. (19)) by setting the argument $a$ of ([42], eq. (19)) to zero. Therefore, the precise closed form of $\Lambda(b, c, g, l)$ should not be summarized in this paper for brevity.

Given the exact closed forms of $\Phi_{\varnothing}$ in (64) and $\Phi_{\mathscr{B}}$ in (70), it is apparent that $\mathscr{H}$ in (61) is solved in a precise closed form. Therefore, $Q_{2 \mathscr{W}}$ in (51) is presented in an exact closed form. Consequently, $\mathbb{Q}_{2}$ in (49) is also presented in an exact closed form.

Because $Q_{1}$ in (29), $Q_{2}$ in (49), and $Q_{3}$ in (34) are all represented in precise closed forms, the SOP of the relay selection in CRNwEH in (27) is found in an exact closed form. The derived SOP formula is helpful to quickly rate the security performance without exhaustive simulations. Upon our knowledge, this formula is novel. Furthermore, some paramount security performance indicators including the PSPMSR and the IP are easily obtained from this formula. To be more specific, the IP indicates the probability of the negative maximum secrecy rate:

$$
\phi=\operatorname{Pr}\left\{C_{\mathrm{sec}}<0\right\}=\mathscr{U}(0) .
$$

Moreover, the PSPMSR indicates the possibility of the strictly positive maximum secrecy rate:

$$
\kappa=\operatorname{Pr}\left\{C_{\mathrm{sec}}>0\right\}=1-\operatorname{Pr}\left\{C_{\mathrm{sec}}<0\right\}=1-\mathcal{U}(0) .
$$

\section{Illustrative Results}

This part presents analytical/simulated results to rate the secrecy performance of the relay selection in $\mathrm{CRNwEH}$ through key specifications where (27) generates analytical

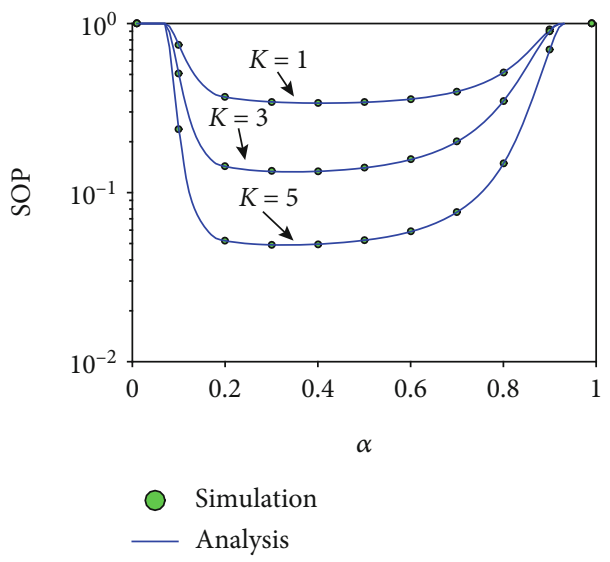

FIgURE 6: SOP versus the time switching ratio.

results, and the computer simulation produces simulated results. Key specifications under consideration are tabulated in Table 1. To limit case studies, equal noise variances, equal power splitting ratios, and equal energy conversion efficiencies are supposed, i.e., $\sigma_{l}^{2}=\sigma_{d}^{2}=\sigma_{w}^{2}=\sigma_{r_{i}}^{2}=\tilde{\sigma}_{r_{i}}^{2}=N_{0}, \eta_{i}=\eta$, $\lambda_{i}=\lambda$ for $i \in[1, K]$.

Figure 3 shows the SOP versus $P_{s m} / N_{0}$ for $\bar{C}_{3}=0.1$ bps $/ \mathrm{Hz}, \alpha=0.6, \varepsilon=0.1, C_{1}=0.2 \mathrm{bps} / \mathrm{Hz}, P_{l} / N_{0}=15 \mathrm{~dB}$, $\lambda=0.8$, and $C_{2}=0.3 \mathrm{bps} / \mathrm{Hz}$. The results confirm the precision of (27) owing to the assortment between the analysis and the simulation. Furthermore, the SOP reduces with the increase in the number of relays ( $K=1$ reduces our system model to relaying transmission (i.e., without relay selection [24-28]) in CRNwEH.). This exposes the effectuality of the relay selection in securing $\mathrm{CRNwEH}$. Moreover, the SOP reduces with the increase in $P_{s m} / N_{0}$. This originates from the truth that the increase in $P_{s m} / N_{0}$ creates $\mathrm{UR}_{i}$ more chances to correctly restore the US's data and to harvest more RF energy from the US's signal, ultimately mitigating the SOP in Stage II. However, the SOP is saturated at large $P_{s m} / N_{0}$. Such saturation comes from the power allotment for US and $\mathrm{UR}_{i}$ in (21) and (22) in which transmission powers of US and $U R_{i}$ do not depend on $P_{s m} / N_{0}$ at high $P_{s m} / N_{0}$ (i.e., the peak transmission power restriction is relaxed at high $\left.P_{s m} / N_{0}\right)$, causing the saturated SOP.

Figure 4 shows the SOP versus $P_{l} / N_{0}$ for $\alpha=0.6, \varepsilon=0.1$, $C_{1}=0.2 \mathrm{bps} / \mathrm{Hz}, P_{s m} / N_{0}=15 \mathrm{~dB}, \bar{C}_{3}=0.1 \mathrm{bps} / \mathrm{Hz}, \lambda=0.8$, and $C_{2}=0.3 \mathrm{bps} / \mathrm{Hz}$. The results corroborate (27) since the simulation coincides with the analysis. Moreover, the SOP reduces with increasing number of relays, again exposing the effectuality of the relay selection in securing $\mathrm{CRNwEH}$. Furthermore, the SOP is minimum at a moderate value of $P_{l}$ for a specific number of relays. Here are reasons for this observation. For low values of $P_{l}$, the interference from LT is small but the unlicensed users must transmit with low power to guarantee the QoS of LR to be fixed at $\varepsilon=0.1$. Therefore, the SOP is large. For high values of $P_{l}$, the interference from LT is large but the unlicensed users must transmit with high power to guarantee the QoS of LR to be fixed at $\varepsilon=0.1$. The increase in the interference from LT may surpass the increase in the unlicensed users' the 


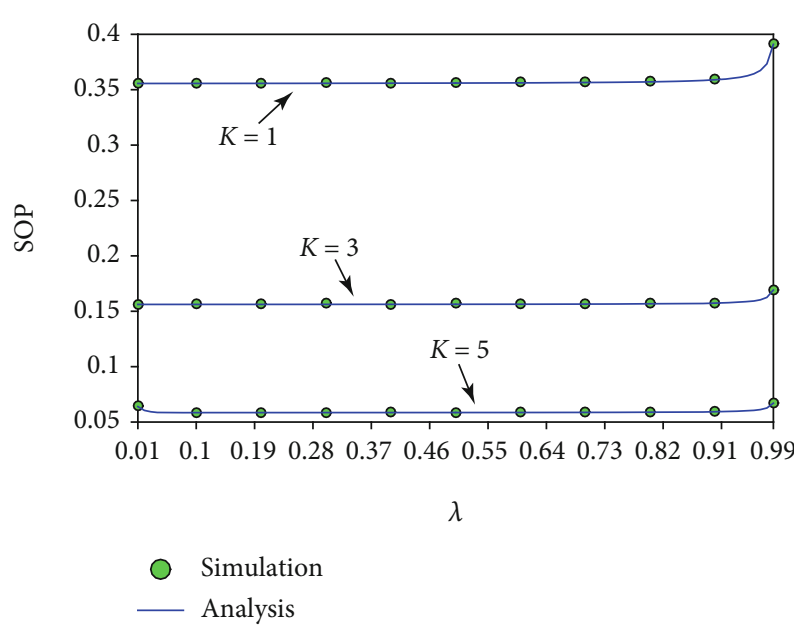

FIgURE 7: SOP versus the power splitting ratio.

transmission power and thus, the SOP is large for high values of $P_{l}$. As such, a moderate value of $P_{l}$ creates the minimum SOP.

Figure 5 shows the SOP versus $\varepsilon$ for $P_{s m} / N_{0}=15 \mathrm{~dB}$, $\alpha=0.6, C_{1}=0.2 \mathrm{bps} / \mathrm{Hz}, P_{l} / N_{0}=17 \mathrm{~dB}, \bar{C}_{3}=0.1 \mathrm{bps} / \mathrm{Hz}$, $\lambda=0.8$, and $C_{2}=0.3 \mathrm{bps} / \mathrm{Hz}$. The results confirm the precision of (27) owing to the assortment between the simulation and the analysis. In addition, the SOP reduces with increasing number of relays as expected. Furthermore, the SOP decreases with increasing the preset outage probability of licensed receiver. The reason is that the increase in $\varepsilon$ permits licensed receivers to tolerate more interference from unlicensed transmitters. Consequently, unlicensed transmitters are able to send signals with larger powers, intimately tempering the outage in Stage II. However, the saturated SOP occurs at large $\varepsilon$ (e.g., $\varepsilon>0.5$ ). The reason for the SOP saturation is the power allotment in (21) and (22) in which the second term in $P_{s}\left(\right.$ or $P_{r_{i}}$ ) does not depend on $\varepsilon$. Consequently, large values of $\varepsilon$ make $P_{s}$ (or $P_{r_{i}}$ ) unchanged, resulting in the saturated SOP.

Figure 6 shows the SOP versus $\alpha$ for $P_{s m} / N_{0}=8 \mathrm{~dB}$, $\varepsilon=0.1, C_{1}=0.2 \mathrm{bps} / \mathrm{Hz}, \bar{C}_{3}=0.1 \mathrm{bps} / \mathrm{Hz}, P_{l} / N_{0}=12 \mathrm{~dB}$, $\lambda=0.8$, and $C_{2}=0.3 \mathrm{bps} / \mathrm{Hz}$. The results verify the accuracy of (27) because the simulation exactly agrees with the analysis. Additionally, the SOP decreases with increasing number of relays as expected. Furthermore, the appropriate selection of $\alpha$, namely, $\alpha_{\text {opt }}$, minimizes the SOP. Here are the reasons for the existence of $\alpha_{\text {opt }}$. Increasing $\alpha$ extends the duration of the Stage I and hence, $\mathrm{UR}_{i}$ scavenges more energy and restores correctly the US's data with a larger possibility. Nonetheless, the increase in $\alpha$ also mitigates the maximum secrecy rate in the Stage II and therefore, the SOP accretes. As a result, the trade-off between the durations of two stages is optimized with $\alpha_{\text {opt }}$ for the minimum SOP.

Figure 7 demonstrates the SOP versus the power splitting ratio $\lambda$ for $\bar{C}_{3}=0.1 \mathrm{bps} / \mathrm{Hz}, P_{s m} / N_{0}=8 \mathrm{~dB}, C_{1}=0.2 \mathrm{bps} / \mathrm{Hz}$, $\alpha=0.6, C_{2}=0.3 \mathrm{bps} / \mathrm{Hz}, P_{l} / N_{0}=12 \mathrm{~dB}$, and $\varepsilon=0.1$. The results expose that the simulation matches the analysis, corroborating (27). In addition, the security capability is enhanced with increasing number of relays as expected. Furthermore, the security performance is optimized by optimally adopting $\lambda$. Here are reasons for the existence

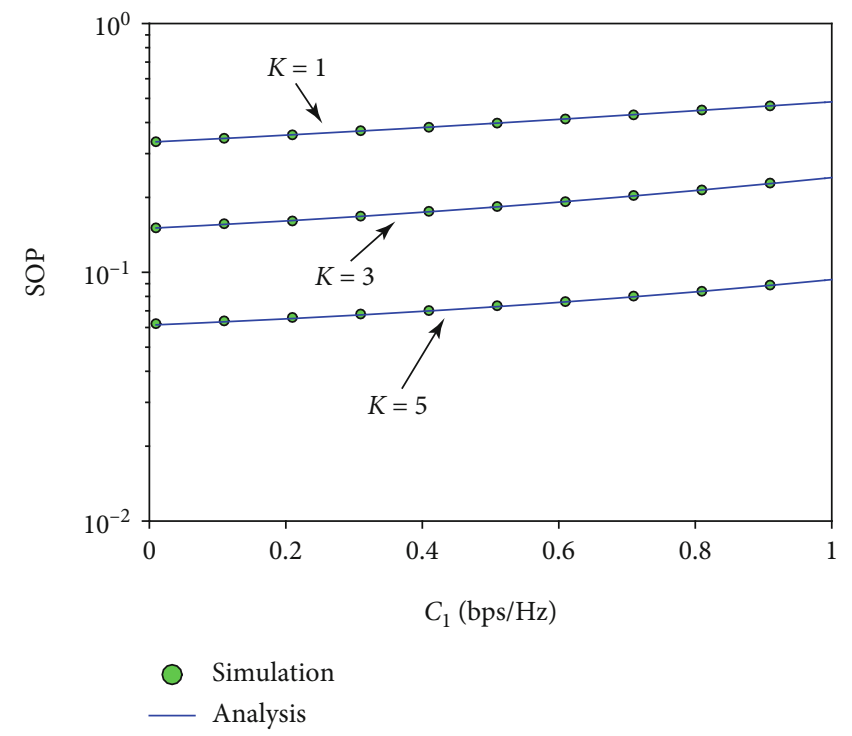

FIGURE 8: SOP versus the target spectral efficiency of unlicensed users.

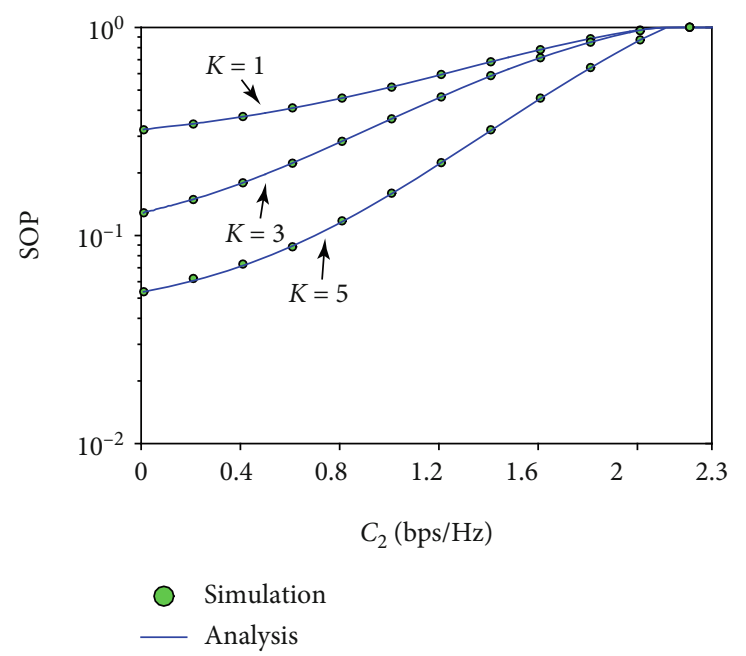

FIGURE 9: SOP versus the target spectral efficiency of licensed users.

of the optimum value of $\lambda$ for the best security performance. The increase in $\lambda$ allows $\mathrm{UR}_{i}$ to harvest more energy and hence, $\mathrm{UR}_{i}$ improves its communication reliability in the Stage II, eventually reducing the SOP. However, the increase in $\lambda$ also mitigates the energy for the message decoder, deteriorating the possibility that $\mathrm{UR}_{i}$ decodes successfully the US's data in the Stage I and inducing more secrecy outage in Stage II. As such, $\lambda$ can be optimally selected to compromise the communication reliability of US and $\mathrm{UR}_{i}$ in both stages.

Figure 8 illustrates the SOP versus $C_{1}$ for $P_{s m} / N_{0}=10 \mathrm{~dB}$, $\lambda=0.8, \alpha=0.6, \bar{C}_{3}=0.1 \mathrm{bps} / \mathrm{Hz}, \varepsilon=0.1, C_{2}=0.3 \mathrm{bps} / \mathrm{Hz}$, and $P_{l} / N_{0}=15 \mathrm{~dB}$. The results show the assortment between the analysis and the simulation, corroborating (27). 


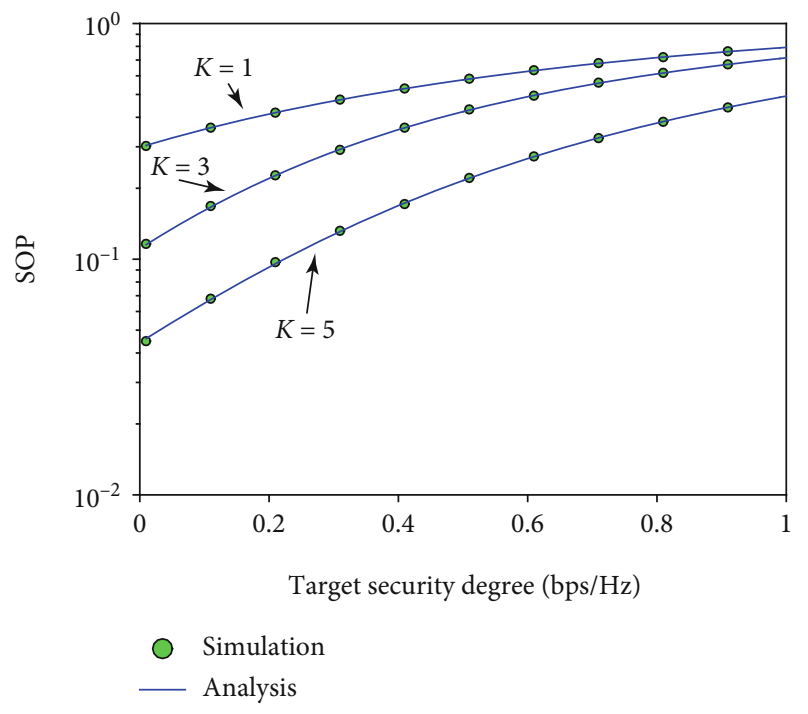

FIGURE 10: SOP versus the target security degree.

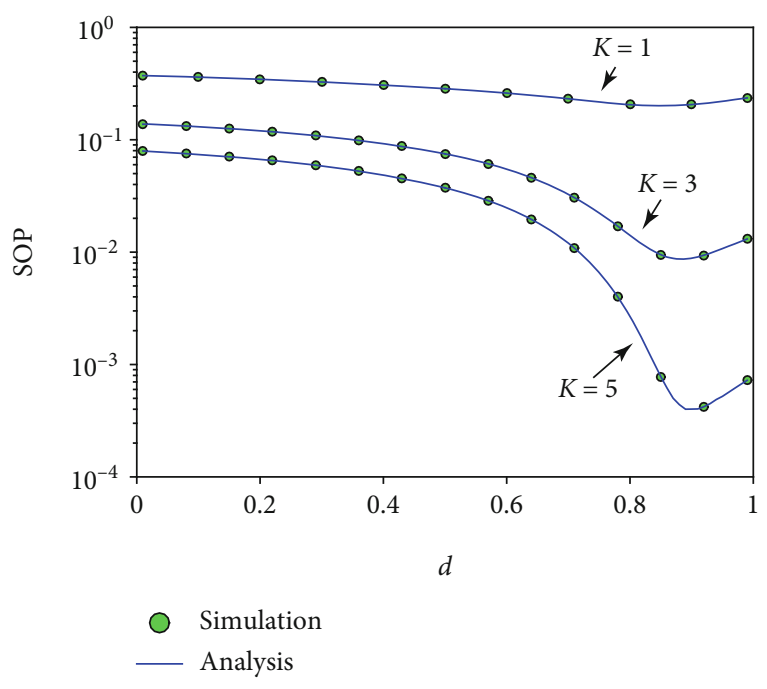

FIGURE 11: SOP versus the relays' positions.

Additionally, the security performance is enhanced with increasing number of relays as expected. Furthermore, the security performance reduces with the increase in $C_{1}$. This is obvious since the higher target spectral efficiency the unlicensed users expect, the lower the possibility for the relays to exactly recover the US's data and thus, the higher the outage probability in Stage II.

Figure 9 demonstrates the SOP versus $C_{2}$ for $P_{s m} / N_{0}=10$ $\mathrm{dB}, \quad \lambda=0.8, \quad \alpha=0.6, \quad \bar{C}_{3}=0.1 \mathrm{bps} / \mathrm{Hz}, \quad \varepsilon=0.1, \quad C_{1}=0.2$ bps $/ \mathrm{Hz}$, and $P_{l} / N_{0}=15 \mathrm{~dB}$. The results verify the agreement between the analysis and the simulation, corroborating the accuracy of (27). Additionally, the SOP reduces with increasing number of relays as expected. Moreover, the security performance is worse with the increase in $C_{2}$. Here are reasons for this. For a certain value of $\varepsilon$ in (17) and (18), the larger target spectral efficiency the licensed users expect, the smaller the interference at licensed users induced by unlicensed users must be and thus, the smaller transmission power the unlicensed users must transmit with, causing the larger SOP. However, the system outage always occurs at high values of $\mathrm{C}_{2}$. Here are the reasons for this. Relied on (21) and (22), we see that $\gamma_{21}$ and $\gamma_{22}\left(\right.$ or $C_{2}$ ) are inversely proportional to the terms inside $[\cdot]^{+}$. Therefore, the increase in $C_{2}$ up to some value (e.g., $2.13 \mathrm{bps} / \mathrm{Hz}$ ) induces [.] ${ }^{+}=0$ and thus, $P_{s}=0$ and $P_{r_{i}}=0$ always happen as $C_{2}$ is above a threshold, inducing the system outage with the probability of 1 .

Figure 10 plots the SOP versus $\bar{C}_{3}$ for $P_{s m} / N_{0}=10$ $\mathrm{dB},=0.6 \lambda=0.8, \varepsilon=0.1, C_{1}=0.2 \mathrm{bps} / \mathrm{Hz}, C_{2}=0.1 \mathrm{bps} / \mathrm{Hz}$, and $P_{l} / N_{0}=15 \mathrm{~dB}$. The results affirm the preciseness of (27) since the simulation agrees with the analysis. Additionally, the security capability is enhanced with increasing number of relays as expected. Moreover, the security performance is worse with the increase in $\bar{C}_{3}$. The reason is since conditioned on specifications, and the higher target security degree causes the higher SOP.

Figure 11 assumes all relays to be located at $(d, 0)$ to demonstrate the SOP versus the relays' positions for $\bar{C}_{3}=0.1$ $\mathrm{bps} / \mathrm{Hz}, P_{s m} / N_{0}=15 \mathrm{~dB}, \varepsilon=0.1, C_{1}=0.2 \mathrm{bps} / \mathrm{Hz}, \alpha=0.6$, $P_{l} / N_{0}=17 \mathrm{~dB}, \lambda=0.8$, and $C_{2}=0.3 \mathrm{bps} / \mathrm{Hz}$. The results corroborate (27) owing to the assortment between the simulation and the analysis. Furthermore, the security performance improves with increasing number of relays, again demonstrating the efficacy of the relay selection in securing $\mathrm{CRNwEH}$. Note that the secrecy outage event occurs as $\mathrm{UR}_{b}$ is unable to exactly restore the US's data (i.e., US is far away from $\mathrm{UR}_{b}$ ) or $\mathrm{UR}_{b}$ is unable to transmit reliably the restored source data to UD (i.e., UD is far away from $\mathrm{UR}_{b}$ ). Consequently, it is natural that the relays' optimum positions always exist. This optimum position optimally compromises the probability that $\mathrm{UR}_{b}$ can precisely decode the US's data with the possibility that $\mathrm{UR}_{b}$ is able to transmit reliably the recovered source data to UD to maximize the security performance. Figure 11 verifies this observation that the best security performance is obtained when $\mathrm{UR}_{b}$ is $d_{\text {opt }}=0.85,0.87,0.91$ away from US for $K=1,3,5$, correspondingly.

\section{Conclusions}

The current paper performed the secrecy performance analysis for the relay selection in CRNwEH by deriving the precise closed-form formula of the SOP under investigation of the licensed outage restriction, Rayleigh fading, the licensed interference, and the peak transmission power restriction. The preciseness of the suggested formula was confirmed by computer simulations. Numerous results illustrated that the relay selection dramatically improves the security capability. Furthermore, the secrecy performance is saturated at large peak transmission power or large target outage probability of licensed user. Additionally, the secrecy performance of the relay selection in CRNwEH depends on several key specifications among which the power splitting ratio, the time switching ratio, and the relays' positions can be optimally adopted to achieve the best information security. 


\section{Appendix}

\section{A. Proof of (30)}

$\mathscr{I}_{i}$ in (29) is rewritten after invoking (5) as

$$
\begin{aligned}
\mathscr{I}_{i} & =\operatorname{Pr}\left\{\gamma_{s r_{i}}<\gamma_{1}\right\}=\operatorname{Pr}\left\{\frac{P_{s}\left|h_{s r_{i}}\right|^{2}}{P_{l}\left|h_{l r_{i}}\right|^{2}+\widehat{\sigma}_{r_{i}}^{2}}<\gamma_{1}\right\} \\
& =\Xi_{\left|h_{l r_{i}}\right|^{2}}\left\{\operatorname{Pr}\left\{\left|h_{s r_{i}}\right|^{2}<\left.\frac{\gamma_{1}}{P_{s}}\left(P_{l}\left|h_{l r_{i}}\right|^{2}+\widehat{\sigma}_{r_{i}}^{2}\right)|| h_{l r_{i}}\right|^{2}\right\}\right\} \\
& =\Xi_{\left|h_{l r_{i}}\right|^{2}}\left\{1-e^{-\frac{\gamma_{1}}{P_{s} \mu_{s r_{i}}}\left(P_{l}\left|h_{l r_{i}}\right|^{2}+\sigma \wedge_{r_{i}}^{2}\right)}\right\} \\
& =1-\int_{0}^{\infty} e^{-\gamma_{1} / P_{s} \mu_{s r_{i}}\left(P_{l} x+\sigma \wedge_{r_{i}}^{2}\right)} \frac{1}{\mu_{l r_{i}}} e^{-x / \mu_{l r_{i}}} d x \\
& =1-\frac{e^{-\sigma \wedge_{r_{i}}^{2} \gamma_{1} / P_{s} \mu_{s r_{i}}}}{\mu_{l r_{i}}\left(\left(P_{l} \gamma_{1} / P_{s} \mu_{s r_{i}}\right)+\left(1 / \mu_{l r_{i}}\right)\right)} .
\end{aligned}
$$

Given $O_{i}$ and $G_{i}$ in (31) and (32), respectively, one can reduce (A.1) to (30), finishing the proof.

\section{B. Proof of (38)}

By considering two scenarios, $\log _{2}\left(\left(1+\gamma_{r_{i} d}\right) /\left(1+\gamma_{r_{i} w}\right)\right) \geq 0$ and $\log _{2}\left(\left(1+\gamma_{r_{i} d}\right) /\left(1+\gamma_{r_{i} w}\right)\right)<0$, one can decompose $\mathscr{G}_{i}$ as

$$
\begin{aligned}
\mathscr{G}_{i} & =\operatorname{Pr}\left\{\log _{2}\left(\frac{1+\gamma_{r_{i} d}}{1+\gamma_{r_{i} w}}\right)<C_{3} \mid \log _{2}\left(\frac{1+\gamma_{r_{i} d}}{1+\gamma_{r_{i} w}}\right)\right. \\
& \left.\geq 0,\left|h_{l d}\right|^{2},\left|h_{l w}\right|^{2}\right\} \times \operatorname{Pr}\left\{\log _{2}\left(\frac{1+\gamma_{r_{i} d}}{1+\gamma_{r_{i}} w}\right)\right. \\
& \left.\geq\left. 0|| h_{l d}\right|^{2},\left|h_{l w}\right|^{2}\right\}+\operatorname{Pr}\left\{0<\left.C_{3}|| h_{l d}\right|^{2},\left|h_{l w}\right|^{2}\right\} \operatorname{Pr}\left\{\log _{2}\left(\frac{1+\gamma_{r_{i} d}}{1+\gamma_{r_{i} w}}\right)\right. \\
& \left.<\left.0|| h_{l d}\right|^{2},\left|h_{l w}\right|^{2}\right\},
\end{aligned}
$$

where $C_{3}$ is given in (37).

Due to the positive target security degree (i.e., $C_{3}>0$ ), (B.1) is simplified as

$$
\begin{aligned}
\mathscr{G}_{i} & =\operatorname{Pr}\left\{\frac{1+\gamma_{r_{i} d}}{1+\gamma_{r_{i} w}}<\left.2^{C_{3}}|| h_{l d}\right|^{2},\left|h_{l w}\right|^{2}\right\} \\
& =\operatorname{Pr}\left\{\gamma_{r_{i} d}<2^{C_{3}}\left(1+\gamma_{r_{i} w}\right)-\left.1|| h_{l d}\right|^{2},\left|h_{l w}\right|^{2}\right\} \\
& =\int_{0}^{\infty} \int_{0}^{2^{C_{3}}(1+x)-1} f_{\gamma_{r_{i} w}, \gamma_{r_{i} d}}\left(x,\left.y|| h_{l d}\right|^{2},\left|h_{l w}\right|^{2}\right) d y d x .
\end{aligned}
$$

Conditioned on $\left|h_{l d}\right|^{2}$ and $\left|h_{l w}\right|^{2}, \gamma_{r_{i} d}$ and $\gamma_{r_{i} w}$ are uncorrelated and hence, their joint PDF is symbolized as the multiplication of their marginal PDFs, i.e., $f_{\gamma_{r_{i} w}, \gamma_{r_{i} d}}\left(x,\left.y|| h_{l d}\right|^{2}\right.$, $\left.\left|h_{l w}\right|^{2}\right)=f_{\gamma_{r_{i} w}}\left(\left.x|| h_{l w}\right|^{2}\right) f_{\gamma_{r_{i} d}}\left(\left.y|| h_{l d}\right|^{2}\right)$. Therefore, (B.2) is further decomposed as

$$
\begin{aligned}
\mathscr{G}_{i} & =\int_{0}^{\infty}\left[\int_{0}^{2^{C_{3}}(1+x)-1} f_{\gamma_{r_{i} d}}\left(\left.y|| h_{l d}\right|^{2}\right) d y\right] f_{\gamma_{r_{i} w}}\left(\left.x|| h_{l w}\right|^{2}\right) d x \\
& =\int_{0}^{\infty} F_{\gamma_{r_{i} d}}\left(2^{C_{3}}[1+x]-\left.1|| h_{l d}\right|^{2}\right) f_{\gamma_{r_{i} w}}\left(\left.x|| h_{l w}\right|^{2}\right) d x .
\end{aligned}
$$

To achieve the closed form for (B.3), the CDF of $\gamma_{r_{i} d}, F_{\gamma_{r_{i} d}}$ $\left(\left.z|| h_{l d}\right|^{2}\right)$, and the PDF of $\gamma_{r_{i} w}, f_{\gamma_{r_{i} w}}\left(\left.z|| h_{l w}\right|^{2}\right)$, are found first.

The CDF of $\gamma_{r_{i} d}$ is

$$
\begin{aligned}
F_{\gamma_{r_{i} d}}\left(\left.z|| h_{l d}\right|^{2}\right) & =\operatorname{Pr}\left\{\gamma_{r_{i} d} \leq\left. z|| h_{l d}\right|^{2}\right\} \\
& =\operatorname{Pr}\left\{\frac{P_{r_{i}}\left|h_{r_{i} d}\right|^{2}}{P_{l}\left|h_{l d}\right|^{2}+\sigma_{d}^{2}} \leq\left. z|| h_{l d}\right|^{2}\right\} \\
& =\operatorname{Pr}\left\{\left|h_{r_{i} d}\right|^{2} \leq\left.\frac{z}{P_{r_{i}}}\left(P_{l}\left|h_{l d}\right|^{2}+\sigma_{d}^{2}\right)|| h_{l d}\right|^{2}\right\} \\
& =1-e^{-z / \mu_{r_{i} d} P_{r_{i}}\left(P_{l}\left|h_{l d}\right|^{2}+\sigma_{d}^{2}\right)} .
\end{aligned}
$$

The PDF of $\gamma_{r_{i} w}$ is straightforwardly obtained from the PDF of $\left|h_{r_{i} w}\right|^{2}$ by using the variable change and hence, the PDF of $\gamma_{r_{i} w}$ can be reduced to

$$
\begin{aligned}
f_{\gamma_{r_{i} w}}\left(\left.z|| h_{l w}\right|^{2}\right) & =\frac{\left(P_{l}\left|h_{l w}\right|^{2}+\sigma_{w}^{2}\right)}{P_{r_{i}}} f_{\left|h_{r_{i}}\right|^{2}}\left(z \frac{P_{l}\left|h_{l w}\right|^{2}+\sigma_{w}^{2}}{P_{r_{i}}}\right) \\
& =\frac{P_{l}\left|h_{l w}\right|^{2}+\sigma_{w}^{2}}{\mu_{r_{i} w} P_{r_{i}}} e^{-z\left(P_{l}\left|h_{l w}\right|^{2}+\sigma_{w}^{2}\right) / \mu_{r_{i}} P_{r_{i}}} .
\end{aligned}
$$

Inserting (B.4) and (B.5) with appropriate variable substitutions into (B.3) results in

$$
\begin{aligned}
\mathscr{G}_{i} & =\int_{0}^{\infty}\left\{1-e^{-\left(2^{C_{3}}[1+x]-1\right) / \mu_{r_{i} d} P_{r_{i}}\left(P_{l}\left|h_{l d}\right|^{2}+\sigma_{d}^{2}\right)}\right\} \frac{\left(P_{l}\left|h_{l w}\right|^{2}+\sigma_{w}^{2}\right)}{\mu_{r_{i} w} P_{r_{i}}} e^{-x\left(P_{l}\left|h_{l w}\right|^{2}+\sigma_{w}^{2}\right) / \mu_{r_{i} w} P_{r_{i}}} d x \\
& =1-\frac{P_{l}\left|h_{l w}\right|^{2}+\sigma_{w}^{2}}{\mu_{r_{i} w} P_{r_{i}}} e^{-\left(2^{C_{3}}-1\right) / \mu_{r_{i} d} P_{r_{i}}\left(P_{l}\left|h_{l d}\right|^{2}+\sigma_{d}^{2}\right)} \int_{0}^{\infty} e^{-\left(2^{C_{3}}\left(P_{l}\left|h_{l d}\right|^{2}+\sigma_{d}^{2}\right) / \mu_{r_{i} d}+\left(P_{l}\left|h_{l w}\right|^{2}+\sigma_{w}^{2}\right) / \mu_{r_{i} w}\right) x / P_{r_{i}}} d x \\
& =1-\frac{P_{l}\left|h_{1 w}\right|^{2}+\sigma_{w}^{2}}{\mu_{r_{i} w} P_{r_{i}}} e^{-\left(2^{C_{3}}-1\right) / \mu_{r_{i} d} P_{r_{i}}\left(P_{l}\left|h_{l d}\right|^{2}+\sigma_{d}^{2}\right)} \frac{P_{r_{i}}}{2^{C_{3}}\left(\left(P_{l}\left|h_{l d}\right|^{2}+\sigma_{d}^{2}\right) / \mu_{r_{i} d}\right)+\left(\left(P_{l}\left|h_{l w}\right|^{2}+\sigma_{w}^{2}\right) / \mu_{r_{i} w}\right)} .
\end{aligned}
$$


Given $A_{i}, B_{i}, C_{i}$, and $D_{i}$ in (39), (40), (41), and (42), respectively, one can shorten (B.6) as (38). This completes the proof.

\section{Data Availability}

The authors declare that all data used to support the findings of this study are included within the article

\section{Conflicts of Interest}

The authors declare that they have no conflicts of interest.

\section{Acknowledgments}

This research is funded by Viet Nam National University Ho Chi Minh City (VNU-HCM) under grant number B2021-2001. We would like to thank Ho Chi Minh City University of Technology (HCMUT), VNU-HCM for the support of time and facilities for this study.

\section{References}

[1] Z. Tian, S. Yi, P. Zhiwen, L. Nan, and Y. Xiaohu, "Matching theory based physical layer secure transmission strategy for cognitive radio networks," IEEE Access, vol. 9, pp. 4620146209, 2021.

[2] K. Ho-van, L. Pham-Hong, S. Vo-Que, and T. Luu-Thanh, "On the performance of opportunistic relay selection in cognitive radio networks with primary user's interference and direct channel," Wireless Personal Communications, vol. 91, no. 1, pp. 345-367, 2016.

[3] K. Ho-Van, "Outage analysis of opportunistic relay selection in underlay cooperative cognitive networks under general operation conditions," IEEE Transactions on Vehicular Technology, vol. 65, no. 10, pp. 8145-8154, 2016.

[4] A. Bletsas, A. Khisti, D. P. Reed, and A. Lippman, "A simple cooperative diversity method based on network path selection," IEEE Journal on Selected Areas in Communications, vol. 24, no. 3, pp. 659-672, 2006.

[5] J. He, S. Guo, F. Wang, and Y. Yang, "Relay selection and outage analysis in cooperative cognitive radio networks with energy harvesting," in 2016 IEEE International Conference on Communications (ICC), pp. 1-6, Kuala Lumpur, Malaysia, 2016.

[6] L. Jiang and H. Tian, "Energy-efficient relay selection scheme for physical layer security in cognitive radio networks," Mathematical Problems in Engineering, vol. 2015, Article ID 587104, 9 pages, 2015.

[7] B. M. ElHalawany, M. Elsabrouty, O. Muta, A. Abdelrahman, and H. Furukawa, "Joint energy-efficient single relay selection and power allocation for analog network coding with three transmission phases," in 2014 IEEE 79th Vehicular Technology Conference (VTC Spring), pp. 1-7, Seoul, Korea, 2014.

[8] H. Lei, H. Zhang, I. S. Ansari et al., "On secrecy outage of relay selection in underlay cognitive radio networks over Nakagami$\mathrm{m}$ fading channels," IEEE Transactions on Cognitive Communications and Networking, vol. 3, no. 4, pp. 614-627, 2017.

[9] C. Wang, H.-M. Wang, and X.-G. Xia, "Hybrid opportunistic relaying and jamming with power allocation for secure cooperative networks," IEEE Transactions on Wireless Communications, vol. 14, no. 2, pp. 589-605, 2015.
[10] H.-M. Wang and X.-G. Xia, "Enhancing wireless secrecy via cooperation: signal design and optimization," IEEE Communications Magazine, vol. 53, no. 12, pp. 47-53, 2015.

[11] X. Li, Q. Wang, M. Liu et al., "Cooperative wireless-powered NOMA relaying for B5G IoT networks with hardware impairments and channel estimation errors," IEEE Internet of Things Journal, vol. 8, no. 7, pp. 5453-5467, 2021.

[12] T.-T. Chan and T.-M. Lok, "Utilizing interference by network coding for simultaneous wireless information and power transfer," IEEE Wireless Communications Letters, 2021.

[13] R. Parekh, U. Shah, and K. George, "Experimental study on 3D fractal base antennas design for efficient Wi-Fi energy harvesting," in 2021 IEEE 11th Annual Computing and Communication Workshop and Conference (CCWC), pp. 1459-1463, NV, USA, 2021.

[14] H. Zheng, K. Xiong, P. Fan, Z. Zhong, and K. B. Letaief, “Age of information-based wireless powered communication networks with selfish charging nodes," IEEE Journal on Selected Areas in Communications, vol. 39, no. 5, pp. 1393-1411, 2021.

[15] K. Ma, Z. Li, P. Liu et al., "Reliability-constrained throughput optimization of industrial wireless sensor networks with energy harvesting relay," IEEE Internet of Things Journal, 2021.

[16] A. D. Wyner, "The wire-tap channel," Bell System Technical Journal, vol. 54, no. 8, pp. 1355-1387, 1975.

[17] L. Ni, X. Da, H. Hu, Y. Yuan, Z. Zhu, and Y. Pan, "Outage-constrained secrecy energy efficiency optimization for CRNs with non-linear energy harvesting," IEEE Access, vol. 7, pp. 175213175221, 2019.

[18] N. Pham-Thi-Dan, T. Do-Dac, K. Ho-Van, S. Vo-Que, and S. Pham-Ngoc, "Effect of Nakagami-m fading on secrecy outage of energy scavenging underlay cognitive networks," in 2019 International Conference on Advanced Technologies for Communications (ATC), pp. 287-291, Hanoi, Vietnam, October 2019.

[19] D. Wang and S. Men, "Secure energy efficiency for NOMA based cognitive radio networks with nonlinear energy harvesting," IEEE Access, vol. 6, pp. 62707-62716, 2018.

[20] F. Wang and X. Zhang, "Secure resource allocation for cooperative cognitive radio networks with dedicated energy sources," in 2018 IEEE International Conference on Communications (ICC), pp. 1-6, Kansas City, MO, USA, May 2018.

[21] H. Lei, M. Xu, I. S. Ansari, G. Pan, K. A. Qaraqe, and M. S. Alouini, "On secure underlay MIMO cognitive radio networks with energy harvesting and transmit antenna selection," IEEE Transactions on Green Communications and Networking, vol. 1, no. 2, pp. 192-203, 2017.

[22] A. Singh, M. R. Bhatnagar, and R. K. Mallik, "Secrecy outage performance of SWIPT cognitive radio network with imperfect CSI," IEEE Access, vol. 8, pp. 3911-3919, 2020.

[23] R. Tan, Y. Gao, H. He, and Y. Cao, "Secrecy performance of cognitive radio sensor networks with an energy-harvesting based eavesdropper and imperfect CSI," in 2018 Asian Hardware Oriented Security and Trust Symposium (AsianHOST), pp. 80-85, Hong Kong, China, December 2018.

[24] N. Pham-Thi-Dan, K. Ho-Van, T. Do-Dac, S. Vo-Que, and S. Pham-Ngoc, "Security analysis for cognitive radio network with energy scavenging capable relay over Nakagami-m fading channels," in 2019 International Symposium on Electrical and Electronics Engineering (ISEE), pp. 68-72, Ho Chi Minh City, Vietnam, October 2019. 
[25] M. Bouabdellah, F. el Bouanani, P. C. Sofotasios et al., "Cooperative energy harvesting cognitive radio networks with spectrum sharing and security constraints," IEEE Access, vol. 7, pp. 173329-173343, 2019.

[26] T. V. Nguyen and B. An, "Cognitive multihop wireless powered relaying networks over Nakagami-m fading channels," IEEE Access, vol. 7, pp. 154600-154616, 2019.

[27] W. Zhao, R. She, and H. Bao, "Security energy efficiency maximization for two-way relay assisted cognitive radio NOMA network with self-interference harvesting," IEEE Access, vol. 7, pp. 74401-74411, 2019.

[28] N. Pham-Thi-Dan, T. Do-Dac, K. Ho-Van, S. Vo-Que, and S. Pham-Ngoc, "On security capability of cooperative communications in energy scavenging cognitive radio networks," in 2019 International Conference on Advanced Technologies for Communications (ATC), pp. 89-93, Hanoi, Vietnam, October 2019.

[29] H. M. Wang, T. X. Zheng, J. Yuan, D. Towsley, and M. H. Lee, "Physical layer security in heterogeneous cellular networks," IEEE Transactions on Communications, vol. 64, no. 3, pp. 1204-1219, 2016.

[30] C. Wang and H. M. Wang, "On the secrecy throughput maximization for MISO cognitive radio network in slow fading channels," IEEE Transactions on Information Forensics and Security, vol. 9, no. 11, pp. 1814-1827, 2014.

[31] H. Lei, R. Gao, K. H. Park, I. S. Ansari, K. J. Kim, and M. S. Alouini, "On secure downlink NOMA systems with outage constraint," IEEE Transactions on Communications, vol. 68, no. 12, pp. 7824-7836, 2020.

[32] X. Li, M. Zhao, M. Zeng et al., "Hardware impaired ambient backscatter NOMA systems: reliability and security," IEEE Transactions on Communications, vol. 69, no. 4, pp. 2723-2736, 2021.

[33] X. Li, M. Zhao, Y. Liu, L. Li, Z. Ding, and A. Nallanathan, "Secrecy analysis of ambient backscatter NOMA systems under I/Q imbalance," IEEE Transactions on Vehicular Technology, vol. 69, no. 10, pp. 12286-12290, 2020.

[34] P. Maji, B. Prasad, S. D. Roy, and S. Kundu, "Secrecy outage of a cognitive radio network with selection of energy harvesting relay and imperfect CSI," Wireless Personal Communications, vol. 100, no. 2, pp. 571-586, 2018.

[35] T. D. Hieu, T. T. Duy, and S. G. Choi, "Performance enhancement for harvest-to-transmit cognitive multi-hop networks with best path selection method under presence of eavesdropper," in 2018 20th International Conference on Advanced Communication Technology (ICACT), pp. 323-328, Chuncheon, Korea (South), February 2018.

[36] K. Ho-van and T. Do-Dac, "Security enhancement for energy harvesting cognitive networks with relay selection," Wireless Communications and Mobile Computing, vol. 2020, Article ID 8867148, 13 pages, 2020.

[37] N. Pham-Thi-Dan, K. Ho-Van, T. Do-Dac, S. Vo-Que, and S. Pham-Ngoc, "Energy harvesting cooperative cognitive networks: relay selection for information security," in 2019 International Symposium on Electrical and Electronics Engineering (ISEE), pp. 93-96, Ho Chi Minh City, Vietnam, October 2019.

[38] K. Ho-Van and T. Do-Dac, "Overlay networks with jamming and energy harvesting: security analysis," Arabian Journal for Science and Engineering, 2021.

[39] P. Nguyen-Huu and K. Ho-van, "Bidirectional relaying with energy harvesting capable relay: outage analysis for Nakagami-m fading," Telecommunication Systems, vol. 69, no. 3, pp. 335-347, 2018.
[40] E. Biglieri, J. Proakis, and S. Shamai, "Fading channels: information-theoretic and communications aspects," IEEE Transactions on Information Theory, vol. 44, no. 6, pp. 26192692, 1998.

[41] I. S. Gradshteyn et al., Table of Integrals, Series and Products, Academic Press, San Diego, CA, USA, 6th edition, 2000.

[42] K. Ho-van and T. Do-Dac, "Reliability-security trade-off analysis of cognitive radio networks with jamming and licensed interference," Wireless Communications and Mobile Computing, vol. 2018, Article ID 5457176, 15 pages, 2018.

[43] K. Ho-Van, "On the performance of maximum ratio combining in cooperative cognitive networks with proactive relay selection under channel information errors," Telecommunication Systems, vol. 65, no. 3, pp. 365-376, 2017. 